\title{
Bakhodir Sidikov*
}

\section{Auf der Suche nach einer verlorenen Zeit? - Visuelle Perspektiven der Kindheit in Mittelasien vor 1917}

DOI 10.1515/asia-2016-0052

Abstract: The following article argues that vintage postcard photographies from Russian Central Asia before 1917 could be read as "conventional" historical sources. The way that provides access to their historical contents beyond the photographers' intentions and no matter how staged the photographies were could be the praxeology, i. e. the conception that vintage postcards images are a manifestation of both local and central social practices in the Central Asian region under Russian rule. Using the example of the debate on childhood in traditional society initiated by French historian Philippe Ariès in his book The Child and Family Life in the Old Régime (1960) the author discusses theoretical and methodological approaches to his topic based on the praxeology.

Keywords: Mittelasien, Kindheit, Praxeologie, Visualität, historische Ansichtskarten

Ушло моё детство, исчезло, пропало, Давно это было, давно ... А может, и вовсе его не бывало И только приснилось оно. ${ }^{1}$

1 „Fortgegangen ist meine Kindheit, verschwunden, verloren //Es war vor langer Zeit, lange her //Vielleicht gab es sie auch gar nicht //Vielleicht habe ich von ihr nur geträumt.“ - Der letzte Vierzeiler, mit dem das Gedicht „Kindheit“ aus der Feder von Mikhail Isakovskij aus dem Jahre 1947-1948 endet. Dieses Gedicht bezieht sich inhaltlich auf die Kindheit eines Knaben (als Lebensalter) aus einer armen russischen Bauernfamilie vor 1917, welche so entbehrungsreich und voll unzumutbarer schwerer körperlicher Arbeit war, dass die Kindheit als eine Schimäre empfunden wird. Isakovskij 1956: 137-139.

*Corresponding author: Bakhodir Sidikov, Universität Bern, Institut für Islamwissenschaft und Neuere Orientalische Philologie, Lerchenweg 36 Bern 3012, Switzerland.

E-mail: bakhodir.sidikov@islam.unibe.ch 


\section{Eine andere - „unglückliche“? - Kindheit}

„Wir bedanken uns beim Genossen Stalin für unsere glückliche Kindheit!“2 (siehe Abbildung 1$)^{3}$ war eine der vielen ideologischen Losungen auf den Transparenten, die in der gesamten Sowjetunion gewöhnlich während der Demonstrationen getragen und an den Häuserfassaden angebracht wurden. Eben jene Parole tauchte wiederum als Refrain in zahlreichen Artikeln der sowjetischen Presse und diversen Fachpublikationen der Zeit auf, welche sich

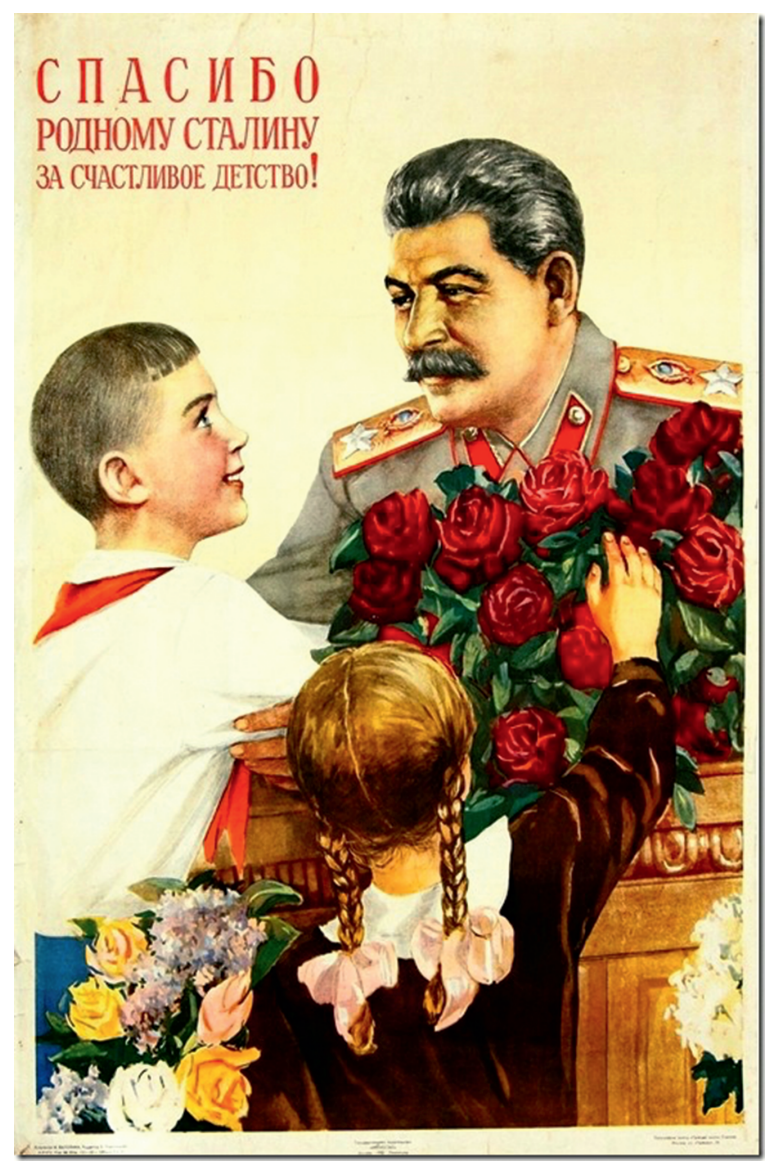

Abbildung 1: Sowjetische Bildpostkarte aus dem Jahre 1939.

2 Russisch: „Спасибо товарищу (oder: родному) Сталину за наше счастливое детство!“ 3 Sowjetische Bildpostkarte aus dem Jahre 1939; aus der Sammlung des Autors. 
dem Thema Kindheit und Jugend widmeten. Dieser Slogan wurde später im Zuge der sog. Entstalinisierung in „Wir bedanken uns bei der Partei für unsere glückliche Kindheit!" ${ }^{4}$ umgewandelt und bestand in dieser Form bis zum Zusammenbruch der Sowjetunion fort.

Die ideologischen Losungen stellen nur in seltenen Fällen eine ausschliesslich ideologische „Lüge“ bzw. die komplette Verkehrung der Wirklichkeit dar. In ihnen wird eine spezifische Verbindung der Ideologie mit der sozialen Welt ersichtlich, und aus dieser Verbindung können - paradoxerweise - auch Rückschlüsse über eben diese soziale Welt gewonnen werden. So ist es auch im Falle der oben zitierten Losung. Neben einer damals selbstverständlichen Huldigung an den grossen Stalin und später an die kommunistische Partei können wir ihr aus der Sicht des heutigen Tages zwei wichtige „Aussagen“ in Bezug auf das Thema „Kindheit“ im Zarenreich bzw. in Mittelasien vor 1917 entnehmen.

Die erste „Aussage“ stellt eine Art stillschweigende Anerkennung dessen dar, dass es unter dem Ancien Régime im Russischen Reich auch eine Kindheit gab. Diese Anerkennung war jedoch für die damaligen politischen Verhältnisse mit ihrer Radikalität beim Bruch mit der Vergangenheit keineswegs selbstverständlich. Denn die Sowjets hätten ja auch behaupten können, dass die Kindheit als gesellschaftliches Phänomen, nicht als spezifisches Lebensalter, im Zarenreich gar nicht existent gewesen wäre. Und solch eine Behauptung wäre auch durchaus berechtigt. Denn die heutige Kindheit auf dem Gebiet der ehemaligen Sowjetunion ist in ihren Grundzügen und ihrer konzeptionellen Ausrichtung erst Ende der 20er - Anfang der 30er Jahre des 20. Jahrhunderts entstanden inklusive der gesamten Infrastruktur der Kinder- und Jugendarbeit und -betreuung (Schwangerschaftsurlaub, Kinder- und Jugendschutzämter, Krippen und Kindergärten, Pionierlager, Pionierpaläste etc.). Konzeptionell und inhaltlich wurde die sowjetische Kindheit überwiegend von Autorinnen und Autoren jüdischer Abstammung herausgearbeitet. Und dies ist kein Zufall, gab es doch in der jüdischen Tradition im Unterschied zur russischen Mehrheitsgesellschaft bis ins kleinste Detail ausgearbeitete Konzepte und durch das religiöse Gesetz sanktionierte Regelwerke im Bereich Kindheit, Jugend und Familie. ${ }^{5}$

4 Russisch: „Спасибо партии (родной) за наше счастливое детство!“

5 Beispielhaft zu nennen sind hier literarische Werke wie „Konduit und Švambranija“ von Lev Kassil'(Kassil' 1935), „Die Kindheit von Marijka“ von Dina Brodskaja (Brodskaja 1938), „Gedichte für Kinder“ von Agnija Barto (Barto 1949), „Als ich klein war“ von Vera Inber (Inber 1954) und „Der Weg führt in die Ferne“ von Aleksandra Bruštejn (Bruštejn 1956), deren Autorinnen und Autoren mit ihrem literarischen Schaffen die sowjetische Kindheit geprägt haben. Vgl. dazu auch Margolina 1992. 
Die zweite „Aussage“ liegt in der Losung selbst begründet und ist für das zu untersuchende Thema noch aufschlussreicher: Ihr zufolge unterschied sich die sowjetische Kindheit qualitativ von der im Zarenreich. „Wie denn auch sonst?“ Bedeutsam ist aber, wie dieser qualitative Unterschied formuliert wurde. Er wird im besagten Slogan durch das gegensätzliche Werturteil „glücklich versus unglücklich“ ebenfalls implizit ausgedrückt.

Diese Losung bzw. das von ihr produzierte ideologische „Kräftefeld“ in der sowjetischen Wissenschaft dürfte massgeblich dazu beigetragen haben, dass während der Sowjetzeit eine ernsthafte Beschäftigung mit der Geschichte der Kindheit im Zarenreich nicht in Erwägung gezogen wurde. Denn sie, die Losung, war auch ein implizit formuliertes, habituell verfestigtes gesellschaftliches Verbot, sich mit der „unglücklichen“ Kindheit zu beschäftigen. Auch nach dem Zusammenbruch der Sowjetunion stand dieses Thema nicht auf der Forschungsagenda, oder es wurde vollkommen an den Rand gedrängt. Denn in der Forschung auf dem in der GUS neu entstandenen internationalen wissenschaftlichen „Markt“ wurden andere, „marktfähigere“ Themenbereiche bevorzugt abgehandelt. So ist die Geschichte der „unglücklichen“ Kindheit im Zarenreich insbesondere an dessen muslimischer Peripherie vor 1917 bis heute ein Desiderat der Forschung geblieben. Grosse Übersichtsarbeiten zum Thema fehlen immer noch; ${ }^{6}$ die vorhandenen Publikationen zu einigen Einzelaspekten stammen fast alle aus der Sowjetzeit und weisen Spuren des Hintergrundes von ideologisierten Staatsapparaten auf.

\section{Die historische Ansichtskarte als potenziell ergiebige Quelle}

Dieser Beitrag versteht sich daher als eine erste Annäherung an die Geschichte der vermeintlich „unglücklichen“ Kindheit im Zarenreich am Beispiel Mittelasiens unter russischer Herrschaft vor 1917. Die zu vollziehende Annäherung erfolgt nicht aus biographischen Notizen und autographischen Quellenmaterialien, ${ }^{7} \mathrm{da}$ diese bereits anderweitig untersucht wurden, sondern exemplarisch aus der Perspektive der russischen Ansichtskarte aus dieser Region. Dabei wird schwerpunktmässig vom Bildmotiv her zu argumentieren sein. Aber wieso gerade diese spezifische, visuelle Quelle? Ich möchte hier auf den hohen

6 Eine Ausnahme bildet das Sammelwerk Rakhimov 1998.

7 Siehe z. B. Ajni 1950; Ajni 1952. 
historiographischen Wert dieses Quellengenres verweisen. Was spricht also für die Heranziehung der russischen Ansichtskarte als historische Quelle zur Erforschung der Geschichte der Kindheit in Mittelasien? Es seien hier nur drei wichtige Hinweise zu geben:

Erstens, wir haben eine beträchtliche Anzahl von historischen Bildpostkarten, wobei das Bildinventar sich in seiner Vielfalt nicht als völlig offener Varianzraum erweist. Die Bildpostkarte generiert durch sich selbst einen Untersuchungsgegenstand und wird so $\mathrm{zu}$ einem historiographischen Unternehmen, in und an dem das kulturelle Gedächtnis im Spannungsfeld von Zentrum und Peripherie untersucht werden kann;

Zweitens: Die allgemeine Quellenlage zur Geschichte der Kindheit der damaligen Zeit in der Region ist auch in materieller Hinsicht recht dürftig. Dies gilt vor allem in Bezug auf die Bekleidung, anhand derer wichtige Erkenntnisse gewonnen werden könnten; ${ }^{8}$

Drittens, die russische Bildpostkarte war ein - wenn nicht das - Massenmedium der betrachteten Zeit, das nicht nur $\mathrm{zu}$ postalischen, sondern auch $\mathrm{zu}$ Sammelzwecken ${ }^{9}$ verwendet wurde (siehe Abbildung 2).

Und viertens, wie die intensiven Sichtungen des dem Autor dieses Beitrages zugänglichen Quellenmaterials aus eigener Ansichtskartensammlung, solchen anderer Sammler und im Internet bereits zeigen, gelangte das Thema „Kind und Kindheit“ in Russisch-Mittelasien so oft auf die Oberfläche dieses Massenmediums, dass wir von einer fast systematischen Beschäftigung der damaligen russischen Fotografen und Produzenten von Ansichtskarten mit dem Thema Kindheit in der Region ausgehen können.

Wir können aus diesem Umstand der gehäuften Thematisierung ableiten, dass in der russischen Gesellschaft, wie sie sich im Zentrum und in den Metropolen findet, ein Bewusstsein für das Thema Kindheit bereits ausgeprägt war. Dies zeugt von einer gewissen „Reife“ der Gesellschaft in Bezug auf den gesamten Komplex „Kind und Kindheit“. Und dieses Bewusstsein fand u.a. in der russischen

8 Siehe z. B. Sukhareva 1979: 7: „ ... Eine Schwierigkeit bei der Erforschung wird durch ein fast vollständiges Fehlen von originalen Materialien komplizierter, deren Studium für die Geschichte der Bekleidung eine Quelle höchster Priorität sein müsste. Aber in Mittelasien gab es keinen Brauch, alte Kleider aufzubewahren. Selbst in den Lagerräumen der Emire von Buchara wurden weder alte Kleider noch die Kleidungsstücke entdeckt, die von der Familie des Emirs getragen wurden; dort wurden hauptsächlich seidene und halbseidene Chalate (eine Art Robe mit langen Ärmeln - B.S.) für Männer aufbewahrt, die wahrscheinlich als Geschenke (Tortuk) geliefert oder zur Beschenkung der Dienerschaft angefertigt wurden (Kleidungssets waren ein Bestandteil der Bezahlung, die von Rang und Status des Beschenkten bestimmt wurden) ... “. Siehe auch Sukhareva 1982.

9 Sommer 1999. 


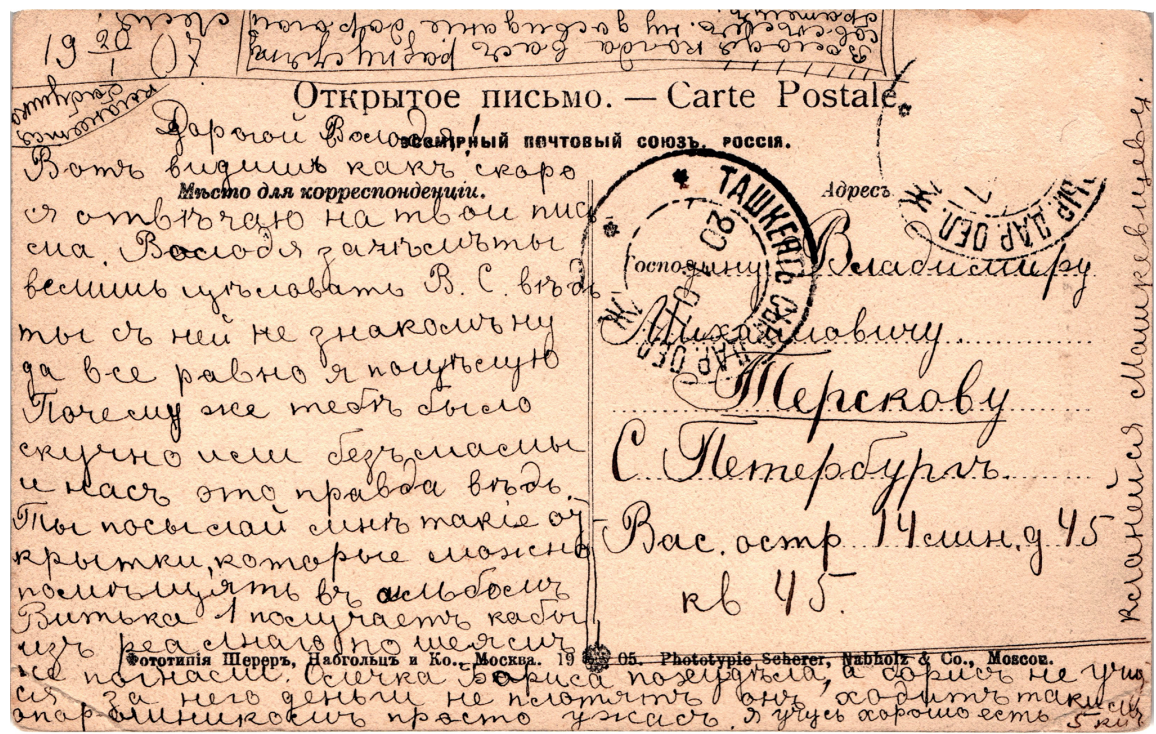

Abbildung 2: Rückseite einer russischen Ansichtskarte, gelaufen am 20.01.1907.

Orientfotografie bzw. ihrer Thematisierung in und durch die Ansichtskarte seinen Niederschlag.

Es wäre daher von grossem historiographischem Nutzen, dieses Massenmedium der damaligen Zeit, welches sich der heutigen Forschung als eine potenziell ergiebige und historiographisch noch nicht erschlossene Quelle anbietet, in Bezug auf verschiedene Themen, insbesondere die Geschichte der vorsowjetischen Kindheit zu befragen.

\section{Wie lässt sich eine visuelle Quelle „zum Sprechen“ bringen?}

Methodisch wäre es unplausibel, eine Quelle, welcher Art auch immer, nicht auch mit anderen Quellen in Verbindung zu bringen. Zudem ist bekannt, dass die Quellenauswertung nur allzu oft unter der mangelnden Offenlegung des theoretischen Rahmens geschieht und die Bewertung der Quelle demnach darunter zu leiden hat. Dies hat zwei Folgen für die die Quellenbetrachtung flankierenden theoretischen Überlegungen, da die isolierte Quellenanalyse a) 
für die Konstruktion und Analyse eines Forschungsgegenstandes (d. h. wenn wir die Quelle „sprechen lassen“ wollen) und b) um das „ganz Grosse“ (d.h. den Gesamtkontext, in diesem Falle die „Geschichte der Kindheit“ in RussischMittelasien) zu erklären, nicht ausreichend sein kann.

Daher muss die Quellenanalyse durch eine erklärende Theorie bzw. Theorien begleitet werden. Die Ambivalenz einer theoretischen Einbettung ist dem Autor durchaus bewusst, denn „... ein und dasselbe Bild kann in sehr verschiedene Theorien integriert werden, woraus folgt, dass sich von ihm aus keine schlagende Evidenz für die eine oder andere Position einstellt, sondern im Gegenteil die theoretische Einbettung allererst ein Bild in einem spezifischen Sinn evident macht."10 Es muss daher auf methodologischer Ebene geklärt werden: Welche Theorien könnten die russische Ansichtskarte, diese spezifische visuelle Quelle, erstens „zum Sprechen“ bringen und zweitens in den grösseren Kontext ihres Gegenstandes, d.h der Kindheit, einbetten? Diese doppelte Aufgabe legt eine mindestens zweifache theoretische Einbindung der visuellen Quelle nahe. Es gilt dabei deutlich zu machen, auf welchen Ebenen der Quelle diese Einbindung ansetzen und ihre erklärende Wirkung entfalten könnte.

Der methodische Vorschlag zum „klassischen“ Umgang mit diesen Fragen besteht darin, dass zunächst die Frage nach der Autorschaft bzw. nach dem Produzenten der Quelle erörtert werden sollte, um sodann die zweite Frage im grösseren Kontext des behandelten Themas „Geschichte der Kindheit“ in Angriff nehmen zu können. Bei der Beschäftigung mit der Frage nach der Autorschaft muss die Bedeutung der Autorschaft jedoch stark relativiert werden. Es kann nicht mehr von dem Autor gesprochen werden. Vielmehr geht es bei der Frage nach dem Autor um die durch diesen wirkenden Dispositionen, die Bourdie als einen „Komplex permanenter Seinsweisen“ bezeichnet (siehe weiter unten).

Machen wir uns dies an unserem Quellenbefund deutlich. Im Falle einer jeden historischen Quelle handelt es sich neben ihren historischen Inhalten vor allem um ihren Autor bzw. Produzenten und zwar nicht so sehr im Sinne einer konkreten dahinterstehenden Person, als vielmehr um dessen Intentionen und die jeweiligen gesellschaftlichen Strukturen, welche - durch den Autor wirkend - zur Entstehung seiner Quelle führen. Allerdings bleibt die Frage nach der Autorschaft in diesem Sinne oft innerhalb der Dichotomie „Intentionen versus Strukturen“ implizit oder explizit gefangen. Diese Dichotomie ist bei der Analyse von Inhalten einer Quelle besonders hinderlich. Denn wir schaffen dadurch, folgt man Bourdieu bei seiner Analyse von künstlerischen Werken, „eine Art Fetisch, die »Autorenintention«, und damit verdinglicht man, fixiert man die Bedeutung des

10 Zittel 2014: 100. 
Werks und entzieht ihm die unendliche Polysemie ... “. ${ }^{11}$ Letztendlich führt die besagte Dichotomie dazu, dass entweder vom „Tod des Autors“ gesprochen oder „aus dem Künstler oder Schriftsteller ein Subjekt seiner Handlungen“12 bzw. der dahinter stehenden grossen gesellschaftlichen Strukturen gemacht wird. ${ }^{13}$

Einen Ausweg aus dieser historiographisch perspektivlosen Dichotomie bzw. ihre Auflösung bieten die Praxistheorien an, und zwar im Rahmen der dispositionalistischen Theorie des französischen Soziologen, in der der Autor bzw. Produzent als „ein agent“ zu begreifen ist. Und dies ist die erste theoretische Einbettung und zwar auf der Ebene der visuellen Quelle selbst. Was würde die Anwendung dieses praxeologisch geprägten Begriffs „agent“ für die Frage nach der Autorschaft bzw. den Produzenten der russischen Ansichtskarten der betrachteten Zeit bedeuten? Überträgt man Bourdieus Theorie auf den russischen Fotografen, so wäre dieser „ein agent, ein Handlungsträger bzw. Akteur, der aufgrund von Dispositionen ${ }^{14}$ handelt, die ihm bewußt oder unbewußt eingeprägt worden sind, und zwar sowohl von Erwachsenen, also von menschlichen Wesen, als auch von seiner Welt, da das Wesentliche der inkorporierten Strukturen jenseits aller ausdrücklichen Erziehung erworben werden konnte, durch den Einfluß von Strukturen. “15

Die Entscheidung für die praxistheoretische (praxeologische) Vorgehensweise bei der Analyse der russischen Ansichtskarte würde nicht ohne weiterreichende Auswirkungen auf das Verständnis der Kindheit im Zusammenhang mit dem betrachteten Thema bleiben. Die Kindheit wird im folgenden Beitrag - praxeologisch reformuliert - als ein besonderer Komplex von sozialen Praktiken innerhalb der Familie und der Gesellschaft definiert, in dessen Zentrum das Kind und sein spezifisches Lebensalter stehen. Diese Praktiken betreffen vor allem den Körper (die Physis) und den Leib (den sozialen Körper) des Kindes, ${ }^{16}$ insbesondere auch eine vorhandene oder fehlende Rücksichtnahme auf die Morphologie des kindlichen

11 Bourdieu 2015: 97.

12 Bourdieu 2015: 97.

13 Vgl. dazu auch Latour 2007.

14 „Die Dispositionen sind jener Komplex permanenter Seinsweisen, die jeder von uns in seinem sozialen Universum erworben hat, das heißt Muster der Wahrnehmung, der Bewertung und des Handelns, die einheitliche und systematische Erzeugungsprinzipien von Praxisformen, von Werken und von Ausdruck im allgemeinen darstellen. Das, was ich Habitus nenne, ist ein System von relativ kohärenten Dispositionen ... “. Bourdieu 2015: 536-537.

15 Bourdieu 2015: 98. Schreibweise von Wörtern mit „ß“ wie im Original der deutschen Übersetzung.

16 Ausführlicher zur Unterscheidung zwischen „Körper“ und „Leib“ in der gegenwärtigen Körpersoziologie siehe z. B. Gugutzer 2015: 13-23. 
Körpers (der Physis und des sozialen Körpers) ${ }^{17}$ bei der Gestaltung des gesellschaftlichen Lebens, die Art und Weise des Kleidens und Bekleidens des Kindes, seine Integration in die Arbeitswelt, eine Nicht-Trennung seiner Welt von der der Erwachsenen, die Gestaltung des Schulwesens etc.

Die zweite theoretische Einbindung erfolgt auf der Ebene des „ganz Grossen“, nämlich der Kindheit, zu welcher die zu analysierende visuelle Quelle einen neuen Zugang schaffen soll. Diese Einbindung wird im Folgenden gestützt durch das kontrovers rezipierte Werk mit dem Titel „Geschichte der Kindheit“ (1960) aus der Feder des französischen Historikers Philippe Ariès. ${ }^{18}$ Diese Einbindung ist - abgesehen von ihrem Hauptziel, einen Zugang zum „ganz Grossen“ zu schaffen - insofern aufschlussreich, als sie es erlaubt, die Gültigkeit von Ariès' Thesen anhand von Quellen aus einer anderen als den in seiner „Geschichte der Kindheit“ betrachteten Regionen zu prüfen. Ariès selbst geht von einem eurozentrischen Blickwinkel aus. Die Thematisierung der Kindheit ausserhalb Europas kommt in seiner Studie nicht vor.

\section{Dreistufiges Analyseverfahren}

Diese zweifache theoretische Einbindung soll im Folgenden in Anlehnung an Bourdieus praxistheoretische Studien von Édouard Manets Gemälde „Frühstück im Grünen“ ${ }^{19}$ in einem dreistufigem Analyseverfahren mit entsprechenden Anpassungen an die historische Ansichtskarte herausgearbeitet und für die Analyse ihrer visuellen Inhalte angewandt werden. Die Übernahme der Bourdieuschen Analyse der impressionistischen Revolution von Manet geschieht nicht zufällig: Die historische Ansichtskarte darf m. E. als ein „legitimes Kind“ des französischen Impressionismus in der zweiten Hälfte des 19. Jahrhunderts und seine Fortsetzung mit anderen - fotografischen - Mitteln betrachtet werden. $^{20}$

17 Die Missachtung der kindlichen Morphologie bzw. die Rücksicht auf diese wird nicht generalisiert. Diese Missachtung bzw. Rücksicht war in der mittelasiatischen Gesellschaft der damaligen Zeit in unterschiedlichem Masse ausgeprägt.

18 Ausführlicher zur Diskussion siehe z. B. Holmes 1968-1969; Berkner 1973; Mclaughlin 1974; DeMause 1974; Shorter 1975; Demaitre 1976-1977; Stone 1977; Agamben 1979; Pollock 1983; Herlihy 1985; Agamben 1993; Habermas 1998; Baumgarten 2004; Gurevič 2009.

19 Bourdieu 2015.

20 „Im Salon von 1867 erreicht die Genremalerei ihren Höhepunkt. Das Bürgertum hatte die Académie als Schiedsrichter über den Geschmack ersetzt, und so mußte die Historienmalerei den Genreszenen weichen. Man rückt von der heroischen Malerei ab, widmet sich der Darstellung kleiner, privater Alltagsszenen. Das wäre im großen ganzen der 
In einem ersten Schritt soll, bevor die eigentliche praxistheoretische „Lektüre“ der russischen Ansichtskarte als historischer Quelle stattfindet, die Figur des Produzenten, des russischen Fotografierenden, soziologisch konstruiert werden. Diese soziologische Konstruktion wird gestützt von „einer sehr seltsamen Übung“ Bourdieus, deren Kernpunkt er als „den Standpunkt des Autors einnehmen“ charakterisierte. ${ }^{21}$ Im Hinblick auf die russische Ansichtskarte gilt es $\mathrm{zu}$ prüfen, welche historisch relevanten Informationen bereits auf der Ebene der „Rekonstruktion der inneren Sicht“ des Fotografen gewonnen werden können. Es handelt sich dabei nicht um den Fotografen im Sinne einer konkreten Person, sondern eines soziologischen Konstruktes: „ ... Das empirische Individuum der Biografen“ ist für die Analyse nach Bourdieu eher hinderlich, da „... die Biografien voller belangloser Informationen schreiben und dabei Wesentliches aus den Augen verlieren ... “22

In einem zweiten Schritt bewegt sich der Analysefokus vom Fotografen auf sein Produkt, die Ansichtskarte. Die Erfahrungen, Wahrnehmungsmuster, -kategorien und -praktiken wie auch Eindrücke von einer visuellen Quelle (die Polysemie des Bildes) können auf Grund vielfältiger Habitusformen und oft konträrer visueller Sozialisierungen der Betrachter, von denen sie ja produziert werden, unterschiedlicher kaum sein. Mit anderen Worten: Es geht um eine historiographische Verarbeitung von visuellen Inhalten der Ansichtskarte und - nicht minder wichtig die visuellen Erfahrungen des heutigen Betrachters mit ihr. Die Einbeziehung des gegenwärtigen Betrachters gehört daher notwendigerweise mit dazu.

Ein Lösungsweg besteht z. B. darin, dass die ausufernde Polysemie einer Bildquelle durch bestimmte Theorieansätze „verwaltet“ und hinsichtlich möglicher Deutungen von historischen Inhalten geordnet wird. Eine Strukturierung bedeutet jedoch keinesfalls, dass die Polysemie in die vertrauten Wahrnehmungsstrukturen eines Betrachters hineingezwängt werden soll. Indem neue und vor allem unterschiedliche Perspektiven versprechende Strukturierungsangebote erarbeitet und angewandt werden, wird die Gefahr einer reduktionistischen „Aneignung“ einer visuellen Quelle durch einen Betrachter wenn nicht gänzlich gebannt, so doch wenigstens erheblich verringert. Diese Verarbeitung muss strukturiert erfolgen, sonst „... kennt das Spiel »Das erinnert mich an« kaum Grenzen ... “23

Entwicklungsprozeß, wichtig, weil er einige zu der These verleitet, die Impressionisten hätten nichts anderes gemacht, als sich der Mode, dem Mondänen, der Bequemlichkeit zu beugen auch die Mitglieder der Académie werden sich dieses Arguments gegen die Impressionisten bedienen ... " Bourdieu 2015: 460.

21 Bourdieu 2015: 140-147, 587-588.

22 Bourdieu 2015: 531.

23 Bourdieu 2015: 56-57. 
Die Praxeologisierung der russischen Ansichtskarte, d.h. die Auffassung, sie sei Ausdruck alltäglicher sozialer Praktiken, könnte ein solches Strukturierungsangebot sein. Welche Gründe sprechen zugunsten der Praxeologisierung? Gerade die lange Belichtungszeit in der Fotografie gegen Ende des 19. - Anfang des 20. Jahrhunderts legt die Vermutung nahe (und das ist die Hauptannahme dieses Beitrages), dass das Bildmotiv der Ansichtskarte kein spontaner Schnappschuss war. Das Bildmotiv zeigte nämlich nicht nur exotische Seiten des lokalen Lebens im russischen Orient, sondern auch fixierte etwas Wesentlicheres als nur Lokalkolorit und Zufälliges, nämlich routinisierte Handlungen, d. h. soziale Vollzüge vor Ort.

Unter diesen sozialen Vollzügen oder Praktiken wird dabei ein „Zusammenspiel von geübten Körpern, gegenständlichen Artefakten, natürlichen Dingen, Gegebenheiten, sozio-materiellen Infrastrukturen und Rahmungen“ verstanden, welches entlang kollektiv geteilter praktischer Wissensformen stattfindet. $^{24}$ In und auf der russischen Ansichtskarte verbinden sich verschiedene Entitäten - Menschen, Körper, Organismen, Gebäude, Tier- und Pflanzenwelt, Dinge, archäologische Artefakte, Worte, Bilder, praktisches Wissen und praktischer Sinn - zu regelmässig geordneten Arrangements, in denen alle Partizipanten Beziehungen miteinander eingehen, zueinander positioniert werden und dadurch ihre jeweilige Bedeutung erhalten. ${ }^{25}$ Mit anderen Worten: In die historische Ansichtskarte sind sowohl die russischen Praktiken des Sehens als auch unterschiedliche Spuren der historischen Wirklichkeit im damaligen Mittelasien hineingeflossen. Aus diesem Grunde kann von einer „doppelläufigen Hermeneutik“ der Ansichtskarten als historischer Quelle gesprochen werden, die in dieser Hinsicht von der bisherigen Forschung noch nicht aufgearbeitet wurde.

Die Praxeologisierung der Ansichtskarte würde somit dem Forscher bei seiner Arbeit mit der besagten Quelle ermöglichen, praxeologische Analyseinstrumente in historischer Hinsicht anzuwenden und so ihre $\mathrm{zu}$ deutenden Inhalte $\mathrm{zu}$,verwalten“ und zu ordnen. Ein Strukturierungsangebot ist auch für den Historiker selbst von grosser Bedeutung, denn eine Quelle muss einen adäquaten kognitiven Stil bei ihm vorfinden, um ihre Wirkung voll $\mathrm{zu}$ entfalten. ${ }^{26}$

Dieser Weg wird folgendermassen abzuschreiten sein: Die russische Ansichtskarte soll einer „praxeologischen“ Lektüre unterzogen werden. Diese „Lektüre“ wird in einem historischen Kontext in Serie mit ähnlichen Ansichtskarten und in Verbindung mit anderen Quellenarten, vor allem schriftlichen Quellen aus dieser Zeit, stattfinden. Ziel der praxeologischen „Lektüre“ besteht darin, historische Inhalte nun aus der vom Fotografen produzierten

24 Schmidt 2012: 13.

25 Vgl. dazu Schatzki 2002: 55.

26 Baxandall 1980: 110-111. 
Ansichtskarte zu Tage zu fördern. Mit anderen Worten: Es gilt, das Bild auf der Vorderseite der Ansichtskarte durch eine praxeologische „Lektüre“ in die historische Faktizität zu überführen. Gleichwohl, ob eine Fotografie auf der Ansichtskarte inszeniert, eine Atelieraufnahme oder ein Schnappschuss ist, beinhaltet sie diverse Spuren der Vergangenheit und bietet gleichzeitig unterschiedliche zum Teil konträre Zugänge zu ihr an, wie dies in der visuellen Anthropologie bereits erkannt und anerkannt wurde: "From the 1990s onwards, there was a growing sense that such images could be read in a way that went beyond or behind the photographers' (presumed) intentions and instead provided access to historical traces of the peoples depicted. No matter how staged or seemingly artificial, these images recorded points in individual and collective lives in which the subjects were sutured into the anthropological project."27

Bei dieser praxistheoretischen Überführung der historischen Ansichtskarte in eine „lesbare“ historische Quelle besteht ebenso wie bei der Beschreibung und Einschätzung eines Gemäldes die Schwierigkeit darin, dass es „... zu jeder Zeit ziemlich absonderlich ist, eine sprachliche Reaktion auf die komplexen nichtsprachlichen Impulse niederzuschreiben, die Gemälde geben wollen ... “. ${ }^{28}$ Mit anderen Worten: Es sollte uns immer bewusst sein, dass diese Überführung eine Art Reduktion der Komplexität und Polysemie einer visuellen Quelle ist, denn ein Text kann das zu deutende Bild nie vollständig ersetzen. ${ }^{29}$

Und in einem dritten, abschliessenden Schritt sollen durch die Einführung einer zweiten Theorie, in diesem Fall „Geschichte der Kindheit“ von Ariès, die aus den ersten beiden Schritten gewonnenen theoretischen Erkenntnisse und historischen Inhalte zu einem Bild des „ganz Grossen“ vervollständigt werden.

\section{5 „Bedeutungslose Bedeutung“: Analoge Fotografie und historische Ansichtskarte}

Vor dem Analyseverfahren wäre es meiner Ansicht nach angebracht, sich den wichtigsten Unterschied zwischen der analogen Fotografie ${ }^{30}$ und der Ansichtskarte

27 Banks/Vokes 2015: 337.

28 Baxandall 1980: 36.

29 Vgl. Bourdieu 2015: 47: Der Autor spricht diese Problematik in Form eines Konflikts zwischen Kritikern und Malern an: „Die Literaten verhelfen den Malern zur Sprache, weil davon ausgegangen wird, daß sie sich nicht mit Worten auszudrücken wissen.“

30 Geimer über die Notwendigkeit einen neuen Namen für die Digitalfotografie zu finden. Geimer 2014: 98-111. 
als historischer Quelle aus demselben Zeitraum vor Augen zu führen. Das Thema der Bedeutung der Fotografie brachte die amerikanische Kunsthistorikerin Rosalind Krauss auf den Punkt, als sie schrieb: „Die Fotografie leitet eine Unterbrechung in der Autonomie des Zeichens ein. Eine Bedeutungslosigkeit umgibt sie, die nur durch das Hinzufügen eines Textes aufgefüllt werden kann.“31 Diese Verknüpfung von Bild und Text sollte jedoch differenzierter analysiert werden. So argumentiert Silke Betscher, dass „... Bild und Text unterschiedliche Aussagesysteme darstellen, die miteinander jeweils sehr spezifische Beziehungen eingehen, zugleich aber auch unabhängig voneinander bestehen bleiben. Kurz: Das Bild generiert Aussagen auch ohne Text ... “32

Berliner Kunsthistorikerin Birte Kleine-Benne vermag den von Rosalind Kraus angesprochenen Aspekt der Fotografie noch schärfer $\mathrm{zu}$ formulieren: „Die ,Fotografie selbst“ gibt es nicht: Es gibt den Kontext der Fotografie, den Fotografen, die technischen Prozesse, den Betrachter, die Blickrelation, die Historie des Materials selbst (gelebte Ränder), die Bildhistorie und -referenzen, die ideologischen Einschreibungen, die Schrift etc.“33

Diese von ihr aufgezählten Dimensionen machen das „Wesen“ der historischen Ansichtskarte aus und heben somit diese einer jeden separat genommenen Fotografie eigene Bedeutungslosigkeit auf. Denn die Ansichtskarte ist eine gerahmte, d. h. kontextualisierte Fotografie, welche den Fotografen und seinen Verlag, die technischen Prozesse und den technischen Habitus des Fotografen, „den Betrachter, die Blickrelation, die Historie des Materials selbst (gelebte Ränder), die Bildhistorie und -referenzen, die ideologischen Einschreibungen, die Schrift etc. “ inkludiert und dadurch die auf der Vorderseite der Ansichtskarte abgedruckte Fotografie mit zum Teil sehr ambivalenten Bedeutungen auflädt. Aus heutiger Sicht betrachtet, werden auch diese Bedeutungen zu historischen Inhalten. Diese und andere Dimensionen sollen im dreistufigen Analyseverfahren, soweit es möglich ist, mitbedacht werden. Die Bedeutung der Ansichtskarte wird zudem erweitert durch „durch das Hinzufügen eines Textes“, also z. B. einer Bildüber- bzw. -unterschrift auf der Vorder- oder Rückseite und einer Mitteilung auf der Rückseite, sofern im Falle einer gelaufenen Bildpostkarte vorhanden, sowie auch durch andere weiterführende Schriftquellen.

31 Zitiert nach Geimer 2014: 30.

32 Betscher 2014: 66.

33 So Dr. Kleine-Benne in einer E-Mail an den Autor vom 6. Juni 2016. 


\section{Performative Notwendigkeit der historischen Ansichtskarte}

Eine von den besagten Dimensionen separierte Fotografie ist nicht „existent“ und somit bedeutungslos und auch nicht notwendig. Wird sie jedoch auf die Vorderseite einer Ansichtskarte transportiert, gewinnt sie eine Bedeutung, denn es stellt sich dem damaligen wie auch dem heutigen Betrachter dann unweigerlich die Frage: „Wieso war es notwendig, diese Fotografie als eine Ansichtskarte zu drucken und massenweise in Umlauf zu bringen?“ Durch diese Notwendigkeit wird die Ansichtskarte performativ im Bourdieuschen Sinne des Wortes, denn diese Notwendigkeit erfordert dann einen bestimmten deutenden Blick auf die Ansichtskarte: „Ein Werk ist aus sich selbst heraus in dem Sinne performativ, als es verlangt, auf eine bestimmte Weise betrachtet $\mathrm{zu}$ werden, und zwar so, daß dem Betrachter seine Notwendigkeit einleuchtet“. ${ }^{34}$ Diese Notwendigkeit im Bourdieuschen Sinne des Wortes macht den Hauptunterschied zwischen einer historischen Ansichtskarte und einer analogen Fotografie aus.

Die performative Notwendigkeit einer visuellen Quelle findet ihre Entsprechung im literaturwissenschaftlichen Begriff „Rezeptionsvorgabe“: „Rezeption im strengen Wortgebrauch nimmt die Phänomene dokumentierter Textverarbeitung in den Blick und ist folglich in starkem Maße auf Zeugnisse angewiesen, in denen sich Einstellungen und Reaktionen als bedingte Faktoren für die Aufnahme von Texten bekunden. Gleichzeitig aber ist der Text selbst eine 'Rezeptionsvorgabe' und damit ein Wirkungspotenzial, dessen Strukturen Verarbeitungen in Gang setzen und bis zu einem gewissen Grade kontrollieren ... “35 Diese Entsprechung bestätigt u.a. die prinzipielle „Lesbarkeit“ einer visuellen Quelle als historisches Zeugnis. ${ }^{36}$

34 Bourdieu 2015: 260.

35 Iser 1994: I. Ein Bild/Gemälde enthält auch eine „Verpflichtung“ des Betrachters, es aktiv zu deuten. Siehe dazu Baxandall 1980: 47: „So durfte jemand, der intellektuell etwas auf sich hielt, bei der Betrachtung von Pieros Gemälde nicht ganz passiv bleiben; er war verpflichtet, Unterschiede wahrzunehmen.“

36 Vgl. auch bei Baxandall 1980: 185-186: „Ein altes Bild dokumentiert eine visuelle Handlung. Man muß lernen, es zu lesen, genauso wie man lernen muß, einen Text aus einer anderen Kultur zu lesen, selbst wenn man in gewissem Sinne die Sprache kennt: Sprache und bildliche Darstellung sind konventionsgebundene Tätigkeiten ... Wenn man aber richtig auf sie zugeht - das heißt, um der Argumentation willen, auf dem Weg, der in diesem Buch („Die Wirklichkeit der Bilder: Malerei und Erfahrung im Italien des 15. Jahrhunderts“ - B.S.) beschritten wurde -, verwandeln sich die Bilder in Dokumente, die genauso gültig sind wie eine Urkunde oder ein Kirchenbuch ... “. 


\section{Wiederholung „einer seltsamen Übung“ Bourdieus}

Im Folgenden sollen die Grundlagen der besagten „seltsamen Übung“ Bourdieus kurz zusammengefasst und dann auf die russische Ansichtskarte (,Tipy Sartovskikh detej“, dt.: „Typen von sartischen Kindern“, siehe Abbildung 3) ${ }^{37}$ angewandt werden.

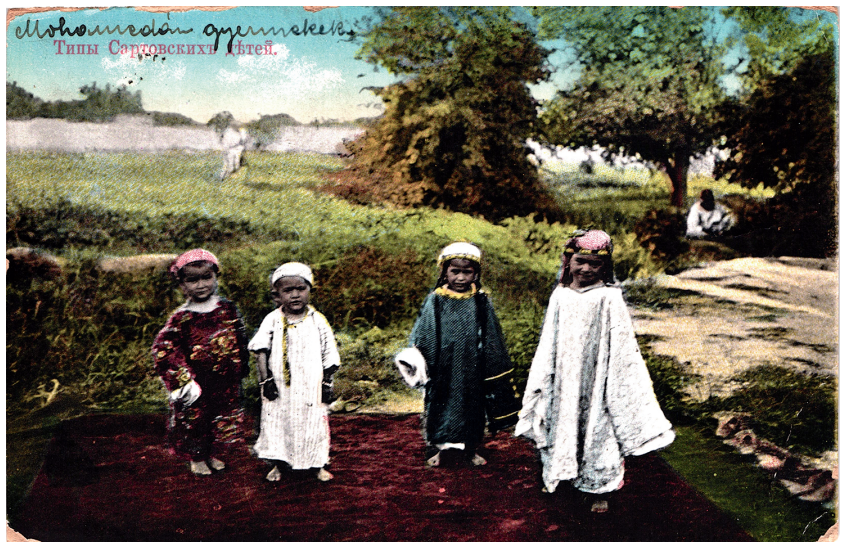

Abbildung 3: Russische Ansichtskarte «Tipy Sartovskikh detej», dt.: „Typen von sartischen Kindern“.

In seiner Analyse des Gemäldes „Frühstück im Grünen“ von Manet schlägt Bourdieu vor, das damals revolutionäre Werk nicht „vom Standpunkt des Betrachters aus, vom Standpunkt des lector, ${ }^{38}$ der sich ein Jahrhundert später nach dem Sinn dieses Bildes fragt ... " $z u$ sehen, sondern zu versuchen, den „Standpunkt des Autors“ einzunehmen. ${ }^{39}$

Bourdieu erklärt, dass „,ich in die Lage des Autors zu versetzen“ „nicht in dem Sinne der einfühlsamen Hermeneuten“ geschehen soll, welche sich „durch eine Art diskursive Identifizierung“ mit dem Schöpfer des Gemäldes gleichsetzen bzw. sich zu diesem machen. Es ist für Bourdieu weder ein anmassendes Psychologisieren noch ein Versuch, sich in den anderen hineinzuversetzen,

37 Aus der Ansichtskartensammlung des Autors.

38 Kursivschreibung im Original.

39 Bourdieu 2015: 140. 
sondern ein Bemühen um die Rekonstruktion des Standpunktes des Autors mit Hilfe folgender Denkoperationen:

Erstens, sich in die historische Lage des Autors versetzen, indem dessen Sozialisation und Habitus thematisiert werden;

Zweitens, sich entsprechendes historisches Wissen über relevante soziale Felder aneignen und

Drittens, die dispositionalistische Theorie der Praxis anwenden. ${ }^{40}$

Welche Folgen hat dies für die Analyse einer Bildquelle? Im Rahmen dieser Theorie verzichtet Bourdieu auf das Begriffspaar „Intention“/,Subjekt“ zugunsten eines anderen: „Disposition“/,Habitus“. In Bezug auf die „Intention“ wird von Bourdieu ein Verzicht zugunsten der Dispositionen vorgeschlagen. In diesem Sinne ist der Maler „ein agent, ein Handlungsträger bzw. Akteur, der nicht von einer Intention geführt wird, sondern ,aufgrund von (seinen - B.S.) Dispositionen handelt, die ihm bewußt oder unbewußt eingeprägt worden sind ... “41 Bourdieu betont dabei: „Der Intention zugunsten der Dispositionen eine Absage erteilen heißt nicht: allen diesen Dingen wie ,Schöpfung‘, ,Intelligenz‘, ,praktische Meisterschaft‘, ,Kunst‘ abschwören - all diesen Dinge, denen wir hohen Wert zusprechen, weil wir ihn uns zusprechen. “42 In Bezug auf das Subjekt postuliert die dispositionalistische Theorie der Praxis ebenfalls einen Verzicht und besagt, dass nicht das „Subjekt der westlichen Tradition“ sein Bild malt, sondern sein Habitus in einem sozialen Feld. ${ }^{43}$ In diesem Sinne ist das Subjekt eines Werkes „eine komplexe Beziehung zwischen einem sozial konstituierten Habitus und einem historisch konstituierten Feld“. Und seine Werke würden, so Bourdieu abschliessend, „in der Beziehung zwischen diesem Habitus und diesem Feld erfunden.“44

In diesem Sinne kann auch eine historische Bildpostkarte als Produkt aus der Beziehung zwischen dem Habitus des russischen Fotografen und dem Feld der russischen Orientfotografie (siehe weiter unten) betrachtet werden. So wie Bourdieu es in Bezug auf das Gemälde von Manet tat, wird im Folgenden am Beispiel der russischen Ansichtskarte „Typen von sartischen ${ }^{45}$ Kindern“ versucht, den Akt des Fotografierens zu rekonstruieren, indem „auf den Blickpunkt eines lector und Hermeneuten“ verzichtet und folglich versucht wird, „das gesellschaftliche Auge“, d.h. die Sicht des Fotografen, soweit es nur möglich ist, zu

40 Bourdieu 2015: 141.

41 Bourdieu 2015: 98.

42 Bourdieu 2015: 126.

43 Bourdieu 2015: 126.

44 Bourdieu 2015: 127.

45 Zur Sartenproblematik s. Abašin 2007. 
rekonstruieren und einzunehmen. Die zu analysierende Ansichtskarte wurde vor 1917 aufgenommen und von dem bekannten Moskauer Handels- und Verlagshaus „Torgovyj Dom Ekkel und Kalakh“ unter der Überschrift „Typen von sartischen Kindern“ gedruckt und in Umlauf gebracht.

\section{Zur Struktur des Bildes}

Die Analyse der Struktur des Bildes ist zwingend erforderlich, denn die Struktur steht in einer sehr spezifischen Verbindung zum Habitus des Fotografen. Diese spezifische Verbindung ermöglicht es, einige Schlüsse auf seinen künstlerischen und kulturellen Habitus zu ziehen, der seine Schaffenskraft im Produktionsfeld entfaltet.

Es handelt sich eindeutig um keine Atelieraufnahme. Das Bild wurde nachträglich koloriert. ${ }^{46}$ Die Farben entsprechen allerdings in hohem Masse den damals und teilweise auch noch heute verwendeten. Es ist zweifelsohne eine Szene, die im Freien entweder auf einem an eine Siedlung angrenzenden Feld oder auf einem Hofland, in einem kleinen Gemüse- und Obstgarten innerhalb einer Siedlung aufgenommen wurde. Die graue Mauer im Hintergrund könnte beides implizieren. Zumindest wird hier eine Begrenzung implizit in das Bildthema hineingewoben.

Im Vordergrund stehen vier im Vergleich mit dem Rest des Bildes scharf dargestellte Kinder auf dunkelrotem, fast purpurfarbenem Boden. Es dürfte sich um einen für Mittelasien typischen einfarbigen Filzteppich handeln. Darauf, dass die Kinder barfuss auf einem Filzteppich stehen, weisen zusätzlich die zur ihrer Linken abgestellten Schuhe hin.

Es könnte sich um zwei Jungen und zwei Mädchen handeln, wobei die Jungen auf der linken Seite und die Mädchen auf der rechten Seite stehen - vermutlich auf Anweisung des Fotografen -, sodass eine Art Geschlechtergegensatz entsteht. Die beiden Mädchen stehen im Verhältnis zu den Jungen enger zusammen; dabei könnten diese beiden Mädchen unterschiedlichen Alters etwas älter sein als die ebenfalls nicht gleichaltrigen Jungen. Die Kinder sind, wie bereits angemerkt, barfuss, sie tragen alle einen Kopfschmuck in Form einer Art Stoffmütze und zudem einteilige Gewänder. Da es sich um eine Aufnahme im Freien und nicht in einem Atelier oder auf einem Fest handelt, ist es eher unwahrscheinlich, dass alle Kinder für eine Aufnahme zurechtgemacht worden sein könnten. D.h. der Fotograf stand auch nicht im „Dienste des Repräsentierten“ (ein Ausdruck von Bourdieu), ${ }^{47}$ wie z. B. die

46 Zur Technik und Geschichte der Kolorierung von Fotografien siehe Antweiler 2013.

47 Bourdieu 2015: 691. 
hier aufgeführte Ansichtskarte („Types de Taschkent. Bai-Batscha“) deutlich veranschaulicht (siehe Abbildung 4). ${ }^{48}$

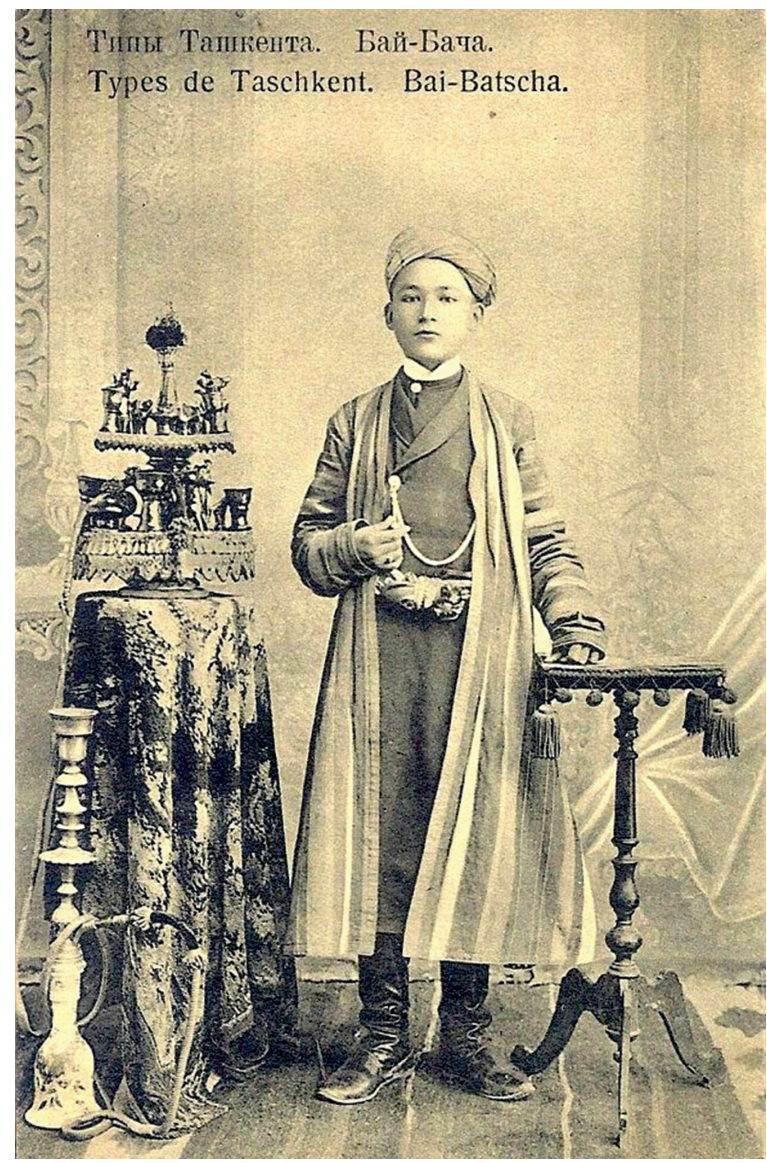

Abbildung 4: Russische Ansichtskarte «Types de Taschkent. Bai-Batscha».

Die Kinder auf der Ansichtskarte „Typen von sartischen Kindern“ tragen offensichtlich Alltagskleidung, d.h. es handelt sich um die alltäglichen Praktiken des Kleidens und Bekleidens von Kindern. Dieser Umstand erhält auf der zweiten Ebene, der praxeologischen „Lektüre“, eine besondere Bedeutung.

48 Aus der Sammlung Nizami Ibraimov, Moskau. 
Die Aufnahme erfolgte wegen der damals üblichen langen Belichtungszeit tagsüber, und zwar während der kalten Jahreszeit, allem Anschein nach im Spätherbst während der Ernte - einer Zeit, wenn die Temperaturen sinken. Dafür spricht vor allem die Bekleidung der Kinder: Damals war es, wie auch heute, üblich, junge Kinder während der warmen Jahreszeit von sehr spärlich bekleidet bis vollständig nackt herumlaufen $\mathrm{zu}$ lassen, wie z. B. der berühmte russische Mittelasienforscher Sergej Nalivkin in seinen Beobachtungen aus dem Ferghanatal anmerkt. ${ }^{49}$

Im direkt dahinter liegenden Grün oder bewachsenen Feld befinden sich zwei hell gekleidete Personen, eine evtl. hockend im Schatten der Bäume und möglicherweise ebenfalls mit Kopfbedeckung sowie eine andere gebückt auf dem Feld bzw. im Grün. Wie zwischen den Bäumen hindurch zu erahnen ist, könnte sich weiter hinten eine weitere, dunkel gekleidete Person befinden. Um wen es sich dabei handelt, muss offen bleiben. Denkbar wären vor allem die Eltern der Kinder oder ihre nächsten Verwandten und Nachbarn, unter derer Obhut sich die Kinder während der Feldarbeit oder einer Abwesenheit der Eltern befinden.

Abgegrenzt wird das Grün bzw. Feld nach hinten durch eine helle, den Bildhintergrund fast vollständig durchziehende Mauer. Nur am rechten Bildrand ist hinter einem der drei Bäume nicht genau $\mathrm{zu}$ erkennen, ob die Mauer dahinter fortläuft. Im gesamten linken Bildhintergrund ist hinter der Mauer unter dem leicht bewölkten Himmel höherer Baumbewuchs zu erkennen.

Links oben im Bereich des Himmels befinden sich in roter Druckschrift mit altem kyrillischem Zeichensatz drei Worte auf Russisch eingefügt: „Типы Сартовскихъ дътей“ (dt.: „Туреn von sartischen Kindern“), die mit einem Punkt abschliessen. Darüber werden in schwarzer Handschrift und in lateinischem Alphabet handschriftlich zwei Worte auf Ungarisch hinzugefügt: „Mohamedán gyerekek“ (dt.: „Mohammedanische Kinder“). Den Angaben auf der Rückseite zufolge ist es eine Bildpostkarte, die von einem österreich-ungarischen Kriegsgefangenen abgeschickt wurde.

\section{Der Titel: Die Texteinheit, die eine Notwendigkeit der Fotografie einleitet}

Bourdieu merkt an, dass bei der Analyse von Bildern ein „Nachdenken“ erforderlich sei, „das von der sozialen Dimension des Titels nicht abstrahiert“. ${ }^{50}$ Daher ist

49 Abašin 2015: 324, siehe auch Šanijazov/Ismailov 1981: 97-101.

50 Bourdieu 2015: 70. 
der Titel der Ansichtskarte in praxeologischer Hinsicht nichts anderes als sprachlicher Ausdruck des technischen und kulturellen Habitus des Fotografen. Der Titel hat eine wichtige einschränkende Funktion, denn er unterbindet ein Phantasieren ins Unendliche. Somit werden auf der betrachteten Ansichtskarte die Kinder auf eine zweifache Weise ins Zentrum der Betrachtung gerückt. Zum einem durch die spezielle fotografische Vorgehensweise: Die Schärfentiefe hebt den Vordergrund hervor, der Hintergrund bleibt unscharf. Diesen Effekt erzielt der Fotograf mit der speziellen Technik der Offenblende, d.h. die Blende ist so weit geöffnet, wie es geht; zum anderen wird das Auge des Betrachters durch den Titel („Typen von sartischen Kindern“) auf die Kinder gerichtet. Der Rest soll verschwommen im Hintergrund bleiben. Des Weiteren bedeutet die Betitelung, dass die Problematik der „bedeutungslosen Bedeutung“ der Fotografie den Produzenten von Ansichtskarten wohl schon damals bewusst war. Und die Betitelung wurde als ein die „bedeutungslose Bedeutung“ der analogen Fotografie aufhebendes Mittel eingesetzt. Denn auch der Titel verleiht der Ansichtskartenfotografie ihre Bedeutung. Und nicht zuletzt stärkt der Titel den performativen Charakter der Fotografie. Er erklärt die Notwendigkeit der Fotografie auf der Vorderseite der Bildpostkarte. Aus dem Titel wird die Notwendigkeit der Fotografie ersichtlich: Es sind sartische, d.h. andere, unbekannte, fremde Kinder, die hier gezeigt werden sollten.

\section{Der Rahmen: Die visuelle Flut ordnen}

Die russische Ansichtskarte jener Zeit misst üblicherweise $8,5 \mathrm{~cm}$ mal $13,5 \mathrm{~cm}$ mit leichten Abweichungen (plus/minus 0,5 cm). Diese Abmessungen sind nicht zufällig entstanden, sondern haben eine anthropologische Grundlage. Es war üblich - und diese Methode ist bei einigen Fotografen der „alten Schule“ auch heute noch im Einsatz -, bei der Suche nach einem passenden Sujet für eine Fotografie mit einem durch die beiden Hände gebildeten Rahmen aus einer schier grenzlosen Masse von visuellen Eindrücken ein bedeutungsvolles Bildmotiv herauszufiltern und einzufangen (siehe Abbildung 5). ${ }^{51}$ Das kleine Format der Ansichtskarte und besonders ihre Motive rücken die Fotografen in die Nähe der Impressionisten. ${ }^{52}$

51 Copyright Sabit Kurmanbek, Grafiker und Maler, Almaty, Kasachstan.

52 „Nach Isaacson (gemeint ist Isaacson/Bouillon 1980 - B.S.) werden die Impressionisten zu privaten Kleinunternehmen, die kleinformatige, den städtischen Wohnverhältnissen angepaßte Bilder bevorzugen, die folglich Werke herstellen, die dem Geschmack der bürgerlichen Klasse entsprechen, das heißt thematisch: Café, Theater, Boulevard, Pferderennen, Szenen an Flußufern oder auf dem Land usw ... “ Bourdieu 2015: 494-495. 


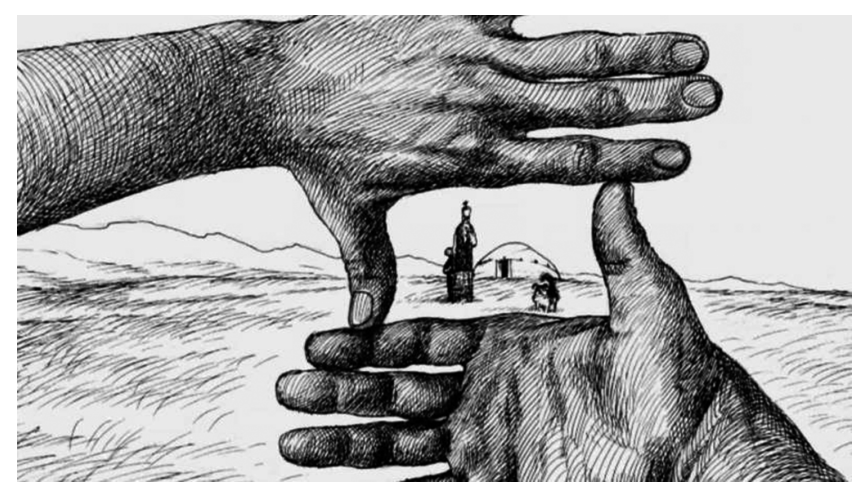

Abbildung 5: Manuelle Suche nach einem passenden Sujet.

Einem Gemälde gleich kommt die Ansichtskarte erst durch den Rahmen zustande, denn „die im Rahmen eingeschlossene Welt wird auf diese Weise zu einer sich selbst genügenden, autarken Welt ... “53 Zugänge zu dieser historischen Welt en miniature werden dann durch die praxeologische Analyse der historischen Ansichtskarte geschaffen.

\section{Zum Habitus des Fotografen}

Im Unterschied $\mathrm{zu}$ Bourdieu, der über seinen Protagonisten (Manet) recht viel wusste, ist über den/die Fotografen/in der obigen Ansichtskarte fast nichts bekannt. Dennoch erscheint es möglich, einiges über seinen Habitus anhand seines Produktes in Erfahrung zu bringen. Die Wahrscheinlichkeit, dass es eher ein Mann als eine Frau war, ist wesentlich höher aufgrund einiger damals vorherrschender Faktoren (Mobilität in einer islamisch geprägten Region, Sicherheitsfragen, Berufswahl als Fotograf etc.). Dadurch hatte der männliche Fotograf mehr Autorität ${ }^{54}$ und „symbolische Gewalt" ${ }^{\text {55 }}$ bei den Einheimischen und konnte in fast all die Bereiche vordringen, die einer Fotografin verschlossen geblieben wären, und die $\mathrm{zu}$ fotografierenden Menschen in eine erwünschte Aufnahme „hineinzwingen“, wobei der Zwang üblicherweise symbolischer Art war. Ein wichtiger Bestandteil seines Habitus ist die gegen Ende des 19. - Anfang des 20. Jahrhunderts weit verbreitete und im Feld der Fotografie eingeschriebene

53 Bourdieu 2015: 385.

54 Zum Konzept der männlichen Herrschaft siehe Bourdieu 2013.

55 Zum Konzept der symbolischen Gewalt siehe Schmidt 2008. 
Auffassung von der Fotografie vor allem als ein Instrument „... der Vermessung und der Dokumentation, bei denen Kategorien wie Autorschaft oder künstlerischer Stil keine Rolle spielten."56 Diese Funktionen der Fotografie (Vermessung und Dokumentation) erklären auch zwei Eigenschaften der historischen Ansichtskarten aus Russisch-Mittelasien:

Erstens: Die Fotografen werden äusserst selten namentlich erwähnt, denn eine Vorstellung vom Copyright war damals zwar vorhanden, hat sich aber nicht feldübergreifend etabliert. Wie im kolonialen Afrika kann davon ausgegangen werden, dass auch die russischen Fotoateliers die Negative aus ihren frühen Aufträgen beibehalten und in der Regel ohne Erlaubnis als Bildpostkarten weiter verwertet haben: "However, in this period the only place in Uganda in which this film could be developed was at Alfred Lobo's Studio, who would then stamp each print they had made with the company stamp. However, more significantly, having developed a print, Lobo's studio would frequently retain its negative, and it is from these amassed negatives that they would later produce many of their picture postcards (although the company did also produce postcards from images they had taken themselves, of course) ... ... This practice of "second printing" to produce postcards was perfectly common throughout Africa during this period. “57

Zweitens: Ansichtskarten mit Fotografien von einer auch für die damaligen technischen Möglichkeiten schlechten und mangelnden Qualität auf der Vorderseite werden dennoch gedruckt und zum Verkauf angeboten. Kein Käufer der Ansichtskarten hat sich daran gestört, denn die schlechte Qualität der Fotografie von damals galt als ein überzeugender Nachweis für ihre „dokumentarische Genauigkeit“. Dieses Verständnis war damals auch in anderen Bereichen verbreitet, die mit Fotografien arbeiteten: „Christeller (Herausgeber eines Atlas der Histotopographie kranker und gesunder Organe - B.S.) war so gefesselt von der Ideologie der Mechanisierung, daß er bestimmt hatte, Mängel auf der Fotografie als eindeutiges Zeichen von Objektivität zu belassen: »Mit Ausnahme der Beseitigung von Fremdeinschlüssen (Staubkörnchen) oder Kratzlinien ist an den Reproduktionen keinerlei Korrektur vorgenommen worden, so dass auch die technisch unvermeidbaren Mängel an manchen Stellen sichtbar sind ... Ich glaubte hierzu verpflichtet zu sein, um zugleich mit grösster Objektivität auch die Grenzen des Verfahrens zu zeigen.«“58

Die dominierende Auffassung des Fotoapparats als ein Instrument „der Vermessung und Dokumentation“ hatte selbstverständlich direkte Auswirkungen auf die Aufnahmepraxis. Sie war nämlich durch ein Streben nach

56 Geimer 2014: 96.

57 Vokes 2015: 397.

58 Daston/Galison 2002: 82. 
„dokumentarischer Genauigkeit“ und einer grösstmöglichen „Treue“ bei der Wiedergabe von aufgenommenen Motiven gekennzeichnet. Es ist ein habitualisiertes und feldübliches Streben, denn eine Eigenwilligkeit (russ.: „otsebjatina“) bzw. künstlerische Freiheit bei der Gestaltung einer Fotografie liesse das Feld der russischen Orientfotografie inkl. der Ansichtskartenproduktion auch nicht zu, wie ein kleiner Exkurs in seine Genese und Zusammensetzung nahelegt.

\section{Orientfotografien in Europa und Russland: Täuschende Namensgleichheit?}

Trotz der Namensgleichheit und ihrer Haupteigenschaft als das Massenkommunikationsmedium dieser Zeit ist die russische Ansichtskarte dennoch vor allem in Bezug auf die Darstellung des Abgebildeten ein ganz anderes Phänomen als ihr westeuropäisches Pendant. Ein Grund dafür war, dass es sich in diesen beiden Fällen um unterschiedliche ikonographische Traditionen handelte. Der wichtigste Unterschied dürfte jedoch darin bestanden haben, dass die russischen Fotografen in ihrem Verständnis (sic!) im Unterschied zu ihren westeuropäischen Kollegen keine Kolonien, sondern als „eigene“59 Landesteile empfundene Gebiete des Russischen Reiches aufgenommen haben. Auch Käufer und Sammler teilten diese Auffassung: So schreibt eine gewisse Ljolja in ihrer Bildpostkarte ${ }^{60}$ aus Taschkent, datiert vom 20.01.1907, abgeschickt und gestempelt am 20.09.1907, an ihren Bruder Volodja in Sankt-Petersburg u. a. Folgendes (siehe Abbildung 2): „Lieber Volodja! ... Du solltest mir solche Ansichtskarten schicken, die in einem Album platziert werden können ... “. Daher ist es nicht zufällig, dass die auf den russischen Ansichtskarten abgebildeten Menschen aus Mittelasien oft als eine Art entfernte, wenn auch etwas exotische, Verwandtschaft präsentiert werden. Diese Eigenschaft verdankt die Bildpostkarte der Fotografie: „Die geographische Versprengtheit der einzelnen Verwandten verlangt gebieterisch die mehr oder weniger regelmäßige Belebung der Verwandtschaftskontakte, und dem genügt die Photographie besser als der bloße Austausch von Briefen."61 Dass Russisch-Mittelasien als Teil der russischen „Grossfamilie“ gesehen wurde, stand in keinem diskursiven Widerspruch zu den kolonialen Bestrebungen Russlands, denn es galt die „unterentwickelten

59 Die Vorstellung, dass die Mittelasiaten entfernte Verwandte seien, scheint in der russischen Gesellschaft von damals verbreitet zu sein.

60 Aus der Ansichtskartensammlung des Autors.

61 Bourdieu 2006: 38. 
Verwandten“ in den neu eroberten Gebieten in der Region durch die russische „Vermittlung“ der europäischen Zivilisation näher zu bringen. ${ }^{62}$

Mit anderen Worten: Ähnlich wie die Fotografien aus einem Familienalbum, haben die Bildpostkarten die Funktion einen „Neuankömmling“ aus RussischMittelasien der „Grossfamilie“ im Kernland Russland vorzustellen und können daher wie die ersteren einer soziologischen „Lektüre“63 unterzogen werden: „Der Austausch von Photos hat den nämlichen Zweck: Mit dem Bildnis präsentiert man dem Ensemble der Gruppe den Neuankömmling, den diese »anerkennen« muß. So ist es denn nur natürlich, daß die Photographie zum Gegenstand einer »Lektüre» wird, die als soziologisch gelten kann, daß sie niemals an sich und für sich, im Hinblick auf ihre technischen oder ästhetischen Qualitäten »gelesen« wird ... “.64

Und „Verwandte“ dokumentiert man bekanntlich anders als „völlig Fremde“: meistens respekt- und würdevoll (Vgl. die französische Ansichtskarte mit der Bildüberschrift auf der Rückseite «Beauté mauresque» mit der russischen Bildpostkarte, die eine sartische Frau zeigt «Sartjanka», dt: „Sartin“ - siehe Abbildung 6 bzw. 7). ${ }^{65}$ Die russischen Fotografen waren daher weitgehend frei vom orientalistisch geprägten tendenziösen Druck bei der Auswahl von Motiven für ihre Aufnahmen. Sie waren ihrem Habitus nach moralisch integere, aufmerksame und „objektive“ Beobachter der exotischen Gebiete ihres eigenen Landes.

Das Übrige tat die Genese des Feldes der Orientfotografie in Russland. Denn das Feld der russischen Orientfotografie, aus dem sich das Feld der Ansichtskartenproduktion in Russland konstituierte, unterschied sich markant vom westeuropäischen bzw. es funktionierte nach anderen „Einsätzen“ und „Spielregeln“ und hatte andere Ziele als in Westeuropa. Die westeuropäischen Fotografen inszenierten in ihren Studios den Orient nach den Vorstellungen der Abendländer: „... In Kairo und Jerusalem etwa stellten sie Szenen aus dem Bazar und dem Harem nach, kleideten dazu die Einheimischen, aber auch die Touristen selbst wie Vertreter einzelner Berufsgruppen, vom Wasserträger bis zum Ziseleur. Als Staffage stellten sie Menschen in faszinierende Landschaften,

62 Vgl. dazu Etkind 2011.

63 Als Gegenbegriff zum „Lesen“, welches als Methode die Bildquelle wie ein Textzeugnis zu interpretieren bestrebt ist, wird in diesem Beitrag der Begriff „Lektüre“ verwendet. Vgl. Bourdieu 2015: 670, Fussnote 35: „Die Metapher des »Lesens« eines Bildes, die in den Universitäten mit der Semiologie erneut in Schwung kam, entspricht vollkommen der akademischen Sicht des Professors als lector. Sie stellt die absolute Antithese zum Gesichtspunkt der Impressionisten und insbesondere Monets dar, für die die künstlerische Wahrnehmung aus Empfindung und Emotion besteht.“

64 Bourdieu 2006: 34.

65 In beiden Fällen wurden oft Prostituierte engagiert (ausführlicher dazu siehe Priščepova 2007: 229) aber eine entblösste Mittelasiatin als Motiv auf einer russischen Ansichtskarte war völlig unvorstellbar. Beide Bildpostkarten aus der Ansichtskartensammlung des Autors. 


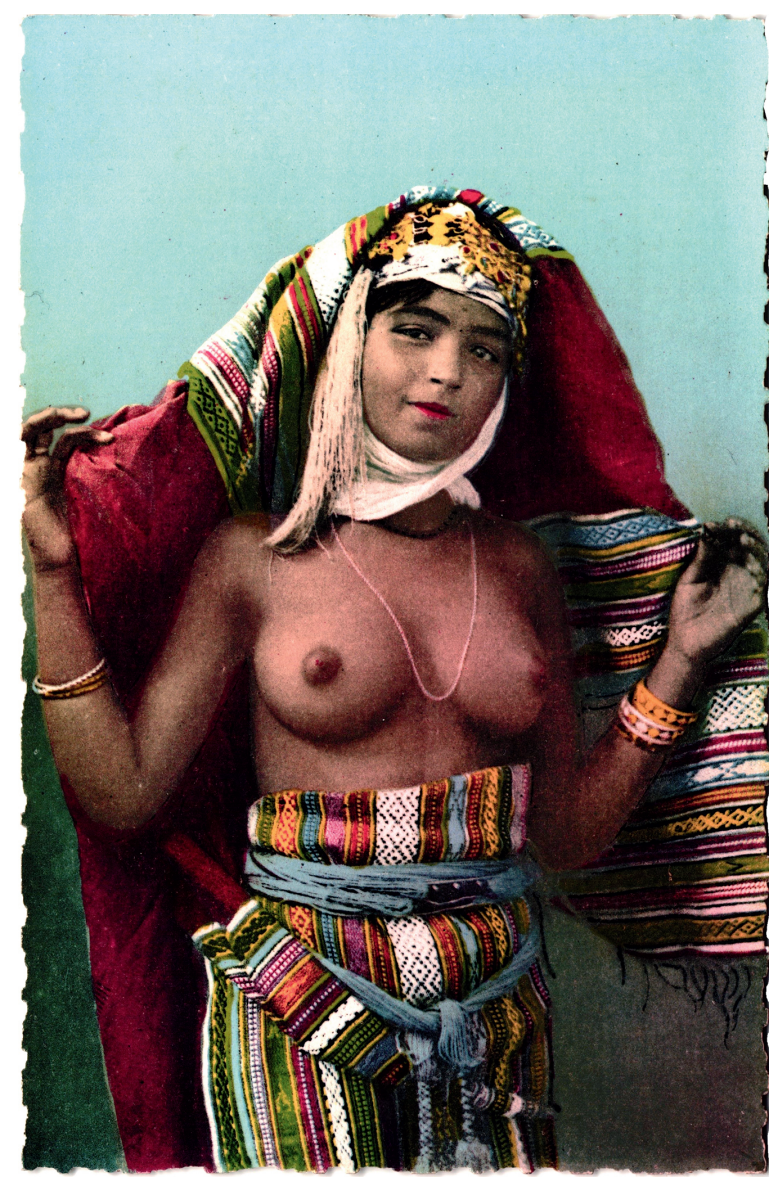

Abbildung 6: Französische Ansichtskarte «Beauté mauresque».

aber auch Kamele, das unvermeidliche Symbol des Orients, oder träge Esel als Zeichen der Rückständigkeit. Die gängigen Stereotypen beeinflussten die kommerziellen Orientaufnahmen.“66

Die russische Orientfotografie hingegen - nur in Teilen privatwirtschaftlich organisiert und kommerziell ausgerichtet wie die westeuropäische - war bestrebt, den „eigenen“ Orient mit „dokumentarischer Genauigkeit“ zu erfassen. Dieses Bestreben erklärt sich weniger durch edle Motive, die in vielen Einzelfällen sicherlich vorhanden waren, sondern vielmehr durch die Genese

66 Hermann 2015; Derenthal 2012; Stiegler/Thürlemann 2015. 


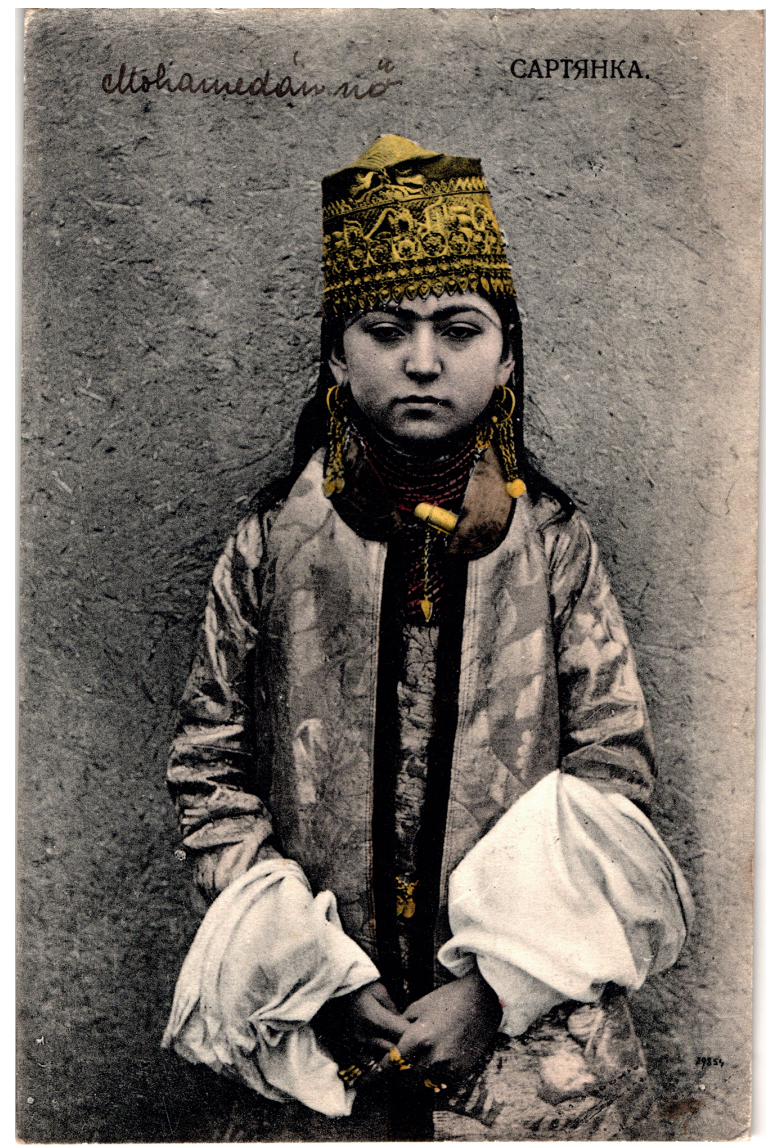

Abbildung 7: Russische Ansichtskarte «Sartjanka», dt: „Sartin“.

und Struktur wie auch den Habitus der Akteure und Institutionen des Feldes der russischen Orientfotografie.

Vor der Entstehung und Verbreitung der Fotografie als Arbeitsinstrument zählten alle russischen Forschungsexpeditionen nach Mittelasien und Sibirien auch die sog. embedded (russ.: otkommandirovannyi) Maler, Zeichner und/oder Grafiker zu ihren Mitgliedern, die von den Organisatoren mit Nachdruck angehalten wurden, im Untersuchungsfeld jene Objekte, Situationen und Ereignisse mit Stift und Aquarellkasten detailreich und vor allen Dingen „realistisch“ und „objektiv“ abzubilden bzw. zu fixieren, welche einem auch noch so aufmerksamen schriftlichen Bericht entgehen könnten. Zahlreiche Maler, Zeichner und Grafiker waren in Mittelasien und Sibirien unterwegs. Diese „Malerei im 
Dienste des Staates“ blieb nicht ohne Folgen für die im Feld der neu entstandenen Orientfotografie geltenden Habitusformen. Mit ihrem Einzug ab 1848 hat das neu konstituierte Feld der russischen Orientfotografie dieses Merkmal oder, genauer gesagt, den Auftrag zur „dokumentarischen Genauigkeit“ von der „Malerei im Dienste des Staates“ „geerbt“. ${ }^{67}$ Dies äusserte sich u. a. auch in der damaligen Bezeichnung der Fotografie - unter dem Namen „Lichtmalerei“ (russ.: svetopis'; risovanie svetom) wurde sie breiten Schichten der russischen Gesellschaft bekannt. ${ }^{68}$

\section{3 „Erfindung unter strukturellem Zwang“: Von der Orientfotografie zur Ansichtskartenproduktion}

Wie fand die Weitertradierung und -wirkung der „dokumentarischen Genauigkeit“ vom Feld der „Dienstmalerei“ in das neue entstandene Feld der russischen Orientfotografie statt? Bourdieu bezeichnet solch einen Prozess der Weitertradierung von Merkmalen eines bestehenden Feldes in ein neues im Entstehen begriffenes als „Erfindung unter strukturellem Zwang“: „In jedem geschichtlichen Moment müssen die Neuankömmlinge mit Produkten der Geschichte rechnen, die in Form von Gebäuden, Bauwerken, Institutionen in die Objektivität eingeschrieben sind - und, wie ich hinzufügen möchte, auch in die Subjektivität in der Form mentaler Strukturen. Gleichzeitig sind die Erfindungen, Neuerungen, Fortschritte, Anpassungen stets Erfindungen unter strukturalem Zwang; das heißt, in jedem Moment ist das Universum der wirklich möglichen Möglichkeiten außerordentlich eng begrenzt, weil bereits in der Vergangenheit getroffene Wahlentscheidungen vorliegen, die in Gestalt objektivierter Zwänge und in Gestalt verinnerlichter, inkorporierter Zwänge existieren. Man steht nicht vor der Alternative Zufall/Notwendigkeit, Freiheit/Notwendigkeit, sondern vor etwas Komplizierterem, das ich in der Formel zusammenfasse: „Erfindung unter strukturalem Zwang«، “69

67 Vgl. Geimer 2009: 96: „Aktueller Anlass dieser Kritik war die von Peter Galassi im Museum of Modern Art kuratierte Ausstellung Before Photography (1981), deren ausdrückliche Absicht es war, die Fotografie innerhalb der Kunstgeschichte zu verorten, ihre Erfindung im frühen 19. Jahrhundert als nicht als diskursiven Einschnitt, sondern als Fortsetzung einer langen Bildtradition zu werten.“

68 Priščepova 2011a: 12.

69 Bourdieu 2014: 245. 
Diese „Erfindung unter strukturalem Zwang“ geschah auch im Feld der russischen Orientfotografie und zwar vor allem personell und institutionell. Personell gab es drei einflussreiche Referenzgruppen, welche die Herausbildung von Habitusformen bei den russischen Fotografen entscheidend bestimmten. Fotograf zu werden, war damals ein Traum vieler in Russland. Am besten geeignet für den Beruf des Fotografen waren auf Grund ihrer Begabung, Erfahrung und des künstlerischen, beruflichen und technischen Habitus wie auch eines entsprechend sozial geschulten Auges verständlicherweise eben die besagten Maler, Zeichner und Grafiker im Dienste des Staates. Vor allem viele Porträtmaler wechselten in den neuen Beruf. Sie bezeichneten sich als „Fotomaler“ (russ.: fotoživopisec). Unter den ersten Fotografen und Fotografinnen, welche die mittelasiatischen und kaukasischen Fotosammlungen der Sankt-Petersburger Kunstkammer (heute: Kunstkammer, Peter-der-Grosse-Museum für Anthropologie und Ethnographie ${ }^{70}$ ) anlegten, waren russische Maler und Malerinnen wie Samuil Dudin, ${ }^{71}$ Ivan Barščevskij, ${ }^{72}$ Nikolai Ščerbina-Kramarenko, ${ }^{73}$ B. F. Romberg, ${ }^{74}$ Antonina Voronina-Utkina ${ }^{75}$ u. a.

70 Website des Museums: http://www.kunstkamera.ru.

71 Samuil Martynovič Dudin (1863-1929) - russischer Maler, Ethnograph, Reisender, Fotograf und Mittelasien- und Sibirienforscher. Ausführlicher zu seinem Lebenslauf und Werk siehe Priščepova 2011b und Uspenskij 2016.

72 Ivan Fedorovič Barščevskij (1851-1948) - „Patriarch“ der russischen Fotografie, der an der Gestaltung von Fotosammlungen der Kunstkamera entscheidend mitwirkte. Ausführlicher zu seinem Lebenslauf und Werk siehe Dlužnevskaja 2011: 30-31. Nicht zu verwechseln mit Lev Semjonovič Barščevskij, einem Namensvetter von ihm (1849-1910) - russischer Oberst polnischer Abstammung und Wissenschaftler, Mitglied der Russischen Geographischen Gesellschaft, Gründer des Museums für Ethnographie in Samarkand; Autor einer einmaligen Fotografiesammlung, die er während seiner Expedition 1876-1897 nach Turkestan anfertigte.

73 Nikolai Ščerbina-Kramarenko (1863-1913) - russischer Maler, Architekt und Mittelasienforscher; in den Jahren 1895, 1896 und 1897 wurde er von der Akademie der Künste nach Mittelasien abkommandiert, um die islamische Architektur der Region zu dokumentieren.

74 „1913 wurde der Maler Boris Fedorovič Romberg nach Buchara abkommandiert, um die dortigen Moscheen zu fotografieren und ethnographische Gegenstände zu sammeln. Er übergab dem Museum (der Kunstkammer - B.S.) eine große Sammlung von Fotonegativen über das Chanat von Buchara, welche jedoch, wie einige Fotodrucke zeigen, schlechter Qualität waren ... Als Maler war er vor allem an den historischen Gebäuden von Schahrisjabs, Alt-Buchara, wie auch an deren Gestaltung und architektonischen Details etc. interessiert ...”. Priščepova 2011a: 109.

75 Antonina Voronina-Utkina (1884-1973) - russische Schullehrerin für Malerei, Malerin, Teilnehmerin der bekannten Grigorij-Potanin-Mittelasienexpedition 1913-1914 (Schwerpunkt: Alltagsgegenstände der kasachischen Stämme). 
Eine weitere, ausserordentlich einflussreiche Referenzgruppe rekrutierte sich aus den Vertretern des in Turkestan stationierten russischen Militärs. Führende Fotografen, die Turkestan fotografisch „dokumentierten“, kamen vor allem aus den Reihen des russischen Militärs. Zu der dritten Gruppe, hier gab es personelle Überschneidungen mit der Gruppe des Militärs (Mittelasien als ans Ausland grenzendes Gebiet wurde als General-Gouvernement - d. h. von den Militärs - verwaltet), gehörten russische Beamte (siehe Abbildung 8, auf dem der damalige Absender anmerkte: „Stadt Taschkent, Puschkin-Strasse, Neubau des Telegrafenamtes, aufgenommen durch einen Beamten-Amateurfotografen“"): ${ }^{76}$

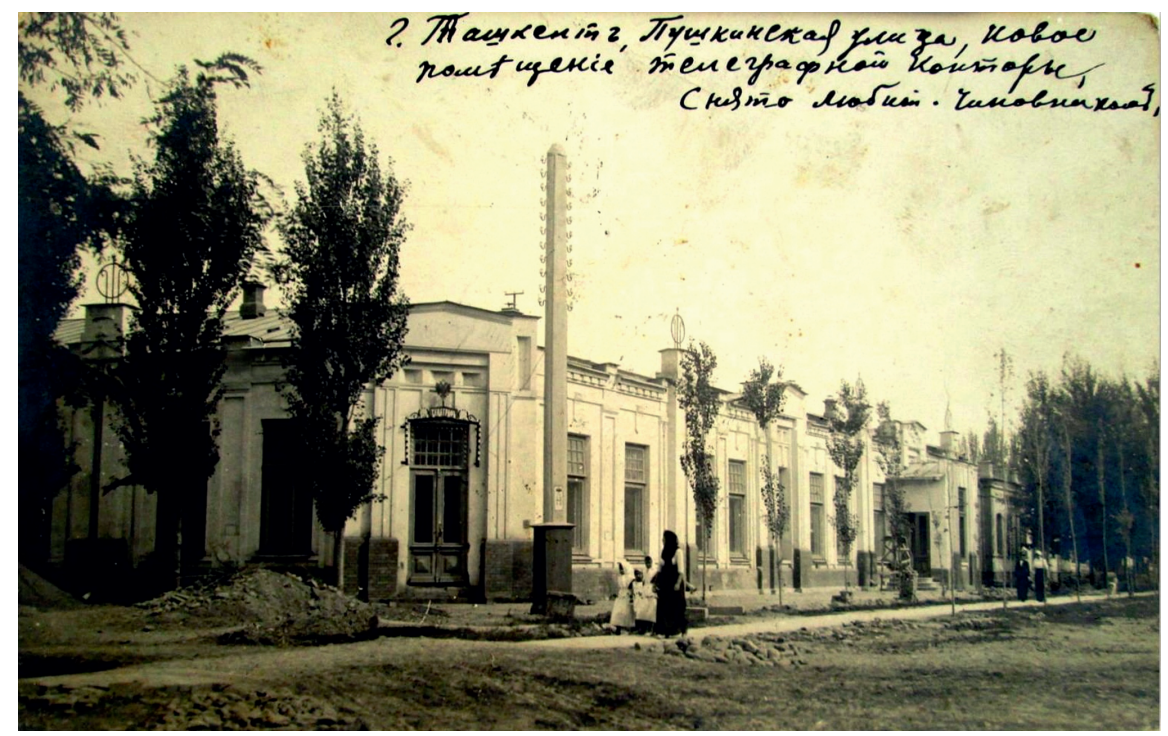

Abbildung 8: Russische Ansichtskarte „Taschkent, Telegrafenamt“.

$\mathrm{Zu}$ den „in die Objektivität eingeschriebenen“ Institutionen gehörten zwei feldbestimmende Institutionen: die bereits erwähnte Sankt-Petersburger Kunstkammer (das erste Museum in Russland), die den Status von Fotografien als Ersatz für fehlende und/oder nicht transportable Exponate betonte und den Mitgliedern ihrer Expeditionen dieses Bewusstsein einschärfte, ${ }^{77}$ und die Kaiserliche Russische Technische Gesellschaft (russ.: „Imperatorskoe Russkoe

76 Freundlicherweise bereitgestellt durch den Moskauer Ansichtskarten-Sammler Boris Mitin. 77 Priščepova 2011a: 24. 
Techničeskoe obščestvo“), deren Tätigkeit vom russischen Militär, das ohnehin an „Präzision“ und „Genauigkeit“ interessiert war, geleitet wurde.

An dieser Stelle ist es zweckdienlich, erneut die Bedeutung einer Fotografie in Gestalt einer Ansichtskarte anzusprechen. Wendet man das praxeologische Verständnis des historischen Dokuments aus der Feder von Bourdieu auf die russische Ansichtskarte von damals an, ${ }^{78}$ dann ist diese eine Art fotografische Stellungnahme, und zwar nicht in einem völlig „leeren Raum“, sondern im sozialen Feld der russischen Orientfotografie mit allen seinen „geerbten“ und neu entwickelten Zwängen und Habitusformen.

Diese fotografische Stellungnahme ist in praxeologischer Hinsicht eine Positionierung im sozialen Feld, die ihre Bedeutung dadurch erhält, dass sie sich einerseits inhaltlich auf ähnliche Positionierungen anderer Fotografen im Feld (d. h. thematisch ähnliche Aufnahmen), andererseits auf das Feld selber, welches Ausdruck dieser Positionierungen ist, bezieht. Dieser zweifache Bezug auf das Feld und ähnliche Produktionen der fotografierenden Kollegen, welcher selbstverständlich nicht immer positiv formuliert wird, ist ein verinnerlichtes „Muss“ als Folge der „Erfindung unter strukturellem Zwang“. Selbst einige zum Feld und den darin enthaltenen Positionen und Positionierungen komplett konträr formulierte Stellungnahmen weisen diesen zweifachen Bezug auf. Diese Zwänge gelten auch für Amateurfotografen: „... Jeder Amateur bezieht sich objektiv, in seiner eigenen Praxis, auf das Bild, das er von der Praxis der anderen hat, sowie auf das Bild, das die anderen von seiner Praxis haben.“79

\section{4 „Stillgestanden!“ oder dokumentarische Inszenierung}

Wir sollten uns durch Worte „dokumentarische Genauigkeit“, „Motivtreue“, „Präzision“ im Habitus der damaligen russischen Fotografen nicht täuschen lassen, denn es handelt sich dabei um ein zeit- und raumgebundenes Verständnis der „dokumentarischen Genauigkeit“, d.h. sie ist gesellschaftlich konstruiert und

78 „Ein Dokument welcher Art auch immer - und das gilt, denke ich, für jedes historische Dokument - ist eine Positionierung in einem Raum, die ihren Sinn bezieht einerseits durch Bezug auf den Raum homologer Positionierungen und andererseits durch Bezug auf den Raum der Positionen, dessen Ausdruck diese Positionierungen sind.“ Bourdieu 2015: 37-38.

79 Bourdieu 2006: 79. 
wird dementsprechend verstanden. ${ }^{80}$ Diese „Motivtreue“ hinderte die russischen Fotografen nicht daran, bestimmte Interventionen und Korrekturen vor, während und nach der Aufnahme vorzunehmen, um dem „Wahrheitsgehalt“ nachzuhelfen bzw. die „Motivtreue“, die „Genauigkeit“ ihrer Fotografien zu potenzieren. Und diese Interventionen und Korrekturen wurden damals nicht als Widerspruch zur oder unzulässiger Eingriff in die „dokumentarische Genauigkeit“ der Fotografie empfunden, ganz im Gegenteil, denn man verhalf somit gewissermassen der „Wahrheit“ zu ihrem Recht, wie die weitere Analyse des Habitus des Fotografen der Ansichtskarte „Typen der sartischen Kinder“ demonstriert.

Der Fotograf oder die Fotografin der hier analysierten Bildpostkarte war gewiss europäischer Herkunft, einen wichtigen Hinweis darauf liefert z. B. die Aufstellung der Kinder. Obwohl es sich um keine Atelieraufnahme handelt, ist es offensichtlich, dass die Kinder vor dem Moment der Aufnahme so nicht herumgestanden haben durften. Entweder spielten sie miteinander, oder sie sassen oder dösten auf dem Filzteppich, oder sie balgten miteinander. Nun treten sie aber in einen „Dialog“ mit dem Fotografen und werden von ihm geordnet. Denn ihre Augen sind auf ihn bzw. seinen Fotoapparat gerichtet. Die Ordnung ist aus technischen Gründen notwendig, denn die herumbalgenden und spielenden Kinder liessen sich schon allein aufgrund der langen Belichtungszeit nicht fotografieren.

Nun erfolgt eine Art Kommando wie „Achtung!“ oder „Stillgestanden!“ an die Kinder - und dem Fotografen gelingt die Aufnahme, welche zum einen die Gesichter im wahrsten Sinne des Wortes dokumentarisch wie für ein amtliches Dokument fixiert (kein Lächeln, keine Grimassen, keine falsche Regung), und es dem Fotografen zum anderen ermöglicht, seinen überwiegend europäischen Kunden das Fremde und Unbekannte in einer vertrauten „Verpackung“ zu liefern bzw. zu verkaufen. Diese „Verpackung“ ist eine Art Reminiszenz an die in der europäischen Malerei „übliche“ Darstellung von Kindern: Die Kinder werden bildkompositorisch in Kontrast gestellt zu den Erwachsenenfiguren im Hintergrund. Der Fotograf lässt diese Konstellation durch die besonderen technischen Darstellungstechniken der Fotografie anklingen: Der Hintergrund ist unscharf aufgenommen und der Fokus auf die Kinder zentriert. Der Fotograf hat kein konkretes europäisches Gemälde im

80 „Wenn man die Photographie für die realistische und objektive Aufzeichnung der sichtbaren Welt hält, dann deshalb, weil man ihr (von Anfang an) gesellschaftliche Gebrauchsweisen eingeschrieben hat, die als >realistisch $<$ und >objektiv< gelten. Sie hat sich mit den äußeren Anzeichen einer $>$ Sprache ohne Regeln und ohne Syntax $<$ dargeboten, d. h. einer $>$ natürlichen Sprache<, weil die Auswahl, die sie im und am Sichtbaren vornimmt, in ihrer Logik ganz und gar der Darstellung der Welt entspricht, wie sie sich in Europa seit dem Quattrocento durchgesetzt hat. Bourdieu 2006: 86. 
Kopf, das in der betrachteten Ansichtskarte präsent ist. Es ist nur ein „generatives

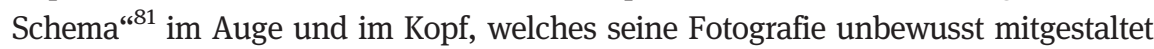
und zur symbolischen Prägnanzbildung mithilft. ${ }^{82}$ Oder mit anderen Worten: In dieser Fotografie findet u.a. sein europäischer kultureller Habitus seinen Niederschlag. Dieser wird erkennbar, wenn man die Komposition der Ansichtskarte mit dem berühmten Gemälde „Las Meninas“ von Diego Velázquez (gemalt 1656) aus dem Madrider Museo del Prado vergleicht (siehe Abbildung 9). ${ }^{83}$

Die Komposition auf der Ansichtskarte bedeutet, dass der Fotograf in einer Fotografie zwei Perspektiven harmonisch vereinigt: einerseits die russischeuropäische Sicht auf die Kinder, andererseits die Spuren der historischen Wirklichkeit in Russisch-Mittelasien. Dabei entsteht kein Widerspruch.

Und das ist nicht alles. Die Ordnung ist auch nach Geschlecht, Alter und Körpergrösse der Kinder erfolgt. Wie bei einem klassischen Gemälde, welches in einem Atelier erschaffen wird, stehen die Kinder für diese Aufnahme Modell: Auf der linken Seite werden zwei Jungen, auf der rechten zwei Mädchen platziert. Eine weitere Ordnung bzw. Struktur ist ebenfalls ersichtlich: Die beiden älteren und körperlich grösseren Kinder, jeweils ein Junge und ein Mädchen, sind an den Flanken des Grüppchens postiert.

Durch die Gruppierung der vier Kinder auf der Ansichtskarte verwandelt der Fotograf das grösste und älteste Mädchen, welches ein besonders übergrosses Gewand trägt, dessen Ärmeln für sie eindeutig zu lang sind, in die Hauptfigur, die den Blick des Betrachters fängt und ihn über die Bedeutung der Fotografie belehrt. Diese vermittelnde Figur war in den religiösen Schauspielen und in der europäischen Malerei des Mittelalters durchaus üblich: „... Zum Beispiel wurden die Spiele von einer Chorfigur eingeleitet, dem festaiuolo, ${ }^{84}$ oft in der Gestalt eines Engels, der während der Handlung des Spiels als Vermittler zwischen dem Betrachter und den

81 Bourdieu 2015: 352-353, 585-586.

82 „... Unter „symbolischer Prägnanz“ soll also die Art verstanden werden, in der ein Wahrnehmungserlebnis, zugleich einen bestimmten nicht-anschaulichen „Sinn“ in sich faßt und ihn zur unmittelbaren konkreten Darstellung bringt. Hier handelt es sich nicht um bloß "perzeptive« Gegebenheiten, denen später irgendwelche "apperzeptive« Akte aufgepfroft wären, durch die sie gedeutet, beurteilt und umgebildet würden. Vielmehr ist es die Wahrnehmung selbst, die kraft ihrer eigenen immanenten Gliederung eine Art von geistiger „Artikulation“ gewinnt - die, als in sich gefügte, auch einer bestimmten Sinnfügung angehört. In ihrer vollen Aktualität, in ihrer Ganzheit und Lebendigkeit, ist sie zugleich ein Leben ,im* Sinn. Sie wird nicht erst nachträglich in diese Sphäre aufgenommen, sondern sie erscheint gewissermaßen als in sie hineingeboren.“ Cassirer 1997: 235.

83 Copyright: „Gemeinfrei“ aus https://de.wikipedia.org/wiki/Las_Meninas.

84 Kursivschreibung im Original. 


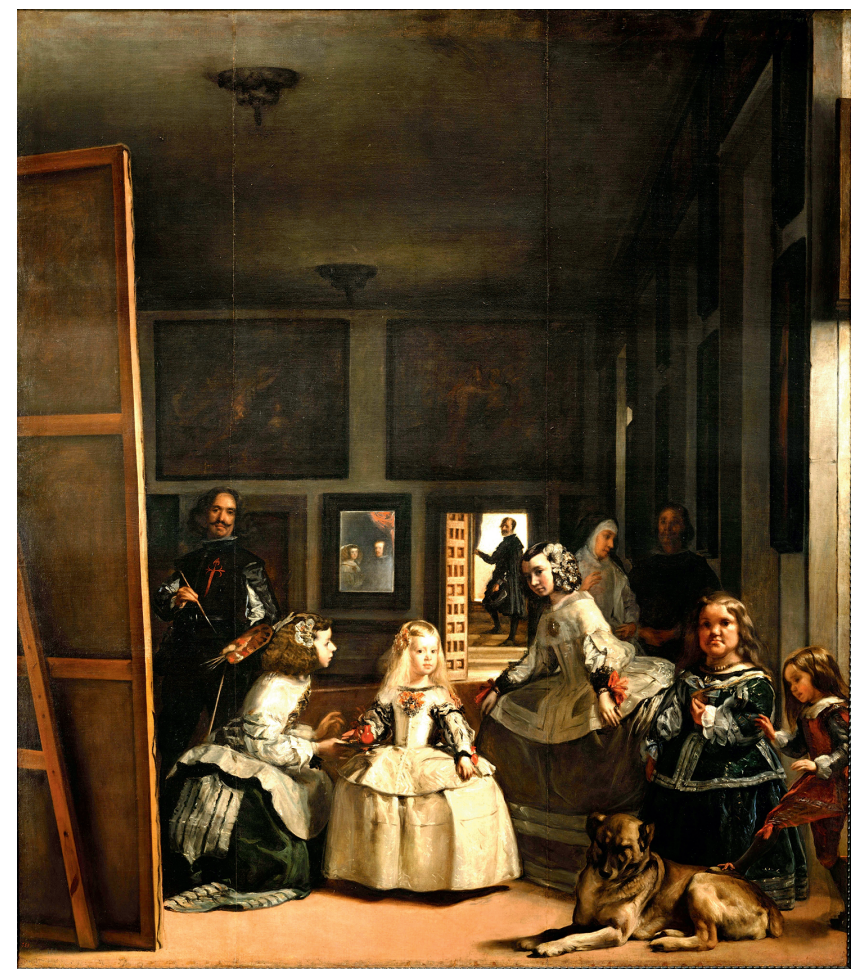

Abbildung 9: „Las Meninas“ („Die Hoffräulein“) von Diego Velaźquez (gemalt 1656).

geschilderten Ereignissen auf der Bühne blieb; ähnliche Chorfiguren, die den Blick fangen und auf die zentrale Handlung hinweisen, wurden oft auch von den Malern verwendet ... Ein mittelalterlicher Autor empfiehlt sie sogar in seiner Schrift unter dem Titel Abhandlung über die Malerei: »Ich wünschte, es gäbe da eine Figur, die uns über das Geschehen des Bildes belehrt und unterrichtet ... $~^{85}$ Diese Art der Gruppierung ist m. E. ein weiterer Hinweis auf den europäisch geprägten Habitus des Fotografen.

Die Eigenschaft des körperlich grössten Mädchens als festaiuolo wird potenziert, wenn die $\mathrm{zu}$ analysierende Ansichtskarte in Serie mit ähnlichen Bildmotiven gebracht wird wie z. B. mit der Abbildung 10 („Deti-bukharcy“, dt.: „Kinder aus Buchara“). ${ }^{86}$

85 Baxandall 1980: 89-90.

86 Aus der Sammlung von Nizami Ibraimov, Moskau. 


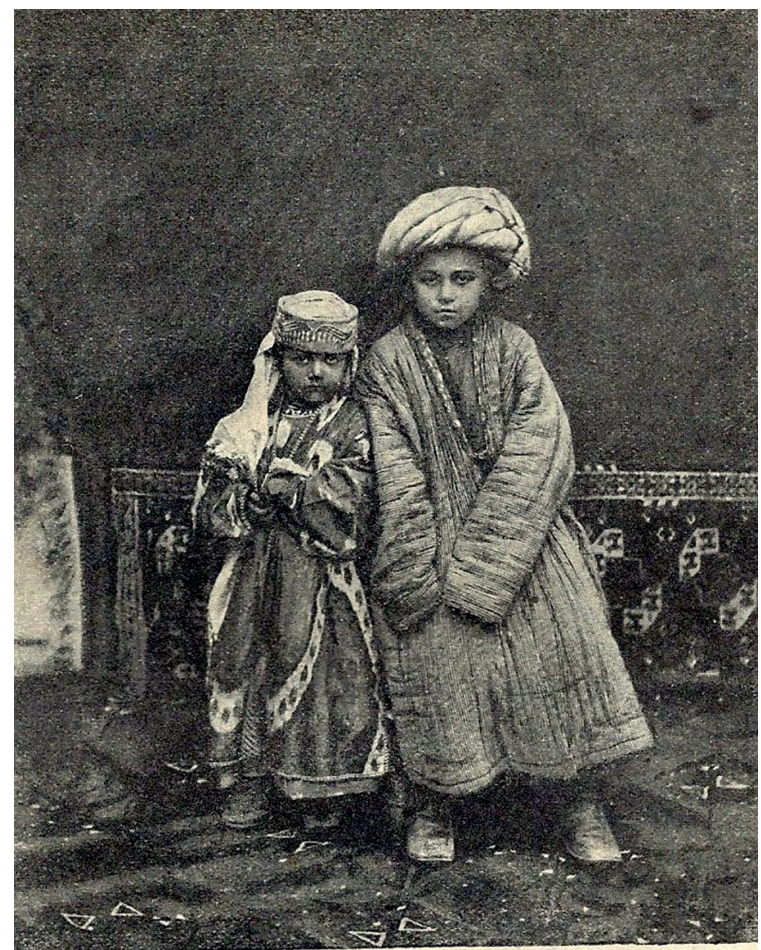

Рис. 197 Дъти-бухарцы.

Abbildung 10: «Deti-bukharcy», dt.: „Kinder aus Buchara“.

\section{Eine „totale soziale Tatsache“}

Bei der Betrachtung der Kindergruppe auf der Ansichtskarte „Typen von sartischen Kindern“ springt eine „Unstimmigkeit““87 ins Auge des Fotografen und auch des heutigen Betrachters: Die Kinder tragen, mit der einzigen Ausnahme des zweiten Jungen von links, besonders übergrosse Gewänder mit für sie zu langen Ärmeln; bei dem Mädchen ganz rechts im weissen Gewand ist diese „Unstimmigkeit“ besonders frappant. Dadurch wird dieses Mädchen zu einer

87 „In Wirklichkeit hält die Photographie einen Aspekt der Realität fest, d.h. das Ergebnis einer willkürlichen Wahl und somit einer Bearbeitung: Von den Eigenschaften des Gegenstandes werden nur jene erfaßt, die in einem besonderen Augenblick und unter einem besonderen Blickwinkel hervortreten ... “ Bourdieu 2006: 85. 
Art des oben erwähnten festaiuolo, also zur Hauptfigur, welche auf die „Haupthandlung“ der Ansichtskarte hinweist.

Diese Eigenschaft scheinen der Fotograf und sein Verlag erkannt zu haben, denn etwas später brachten sie einen Ausschnitt aus der ursprünglichen Fotografie der Kindergruppe als eine neue Schwarzweiss-Ansichtskarte mit demselben Titel („Typen von sartischen Kindern“) in Umlauf (siehe Abbildung 11). ${ }^{88}$

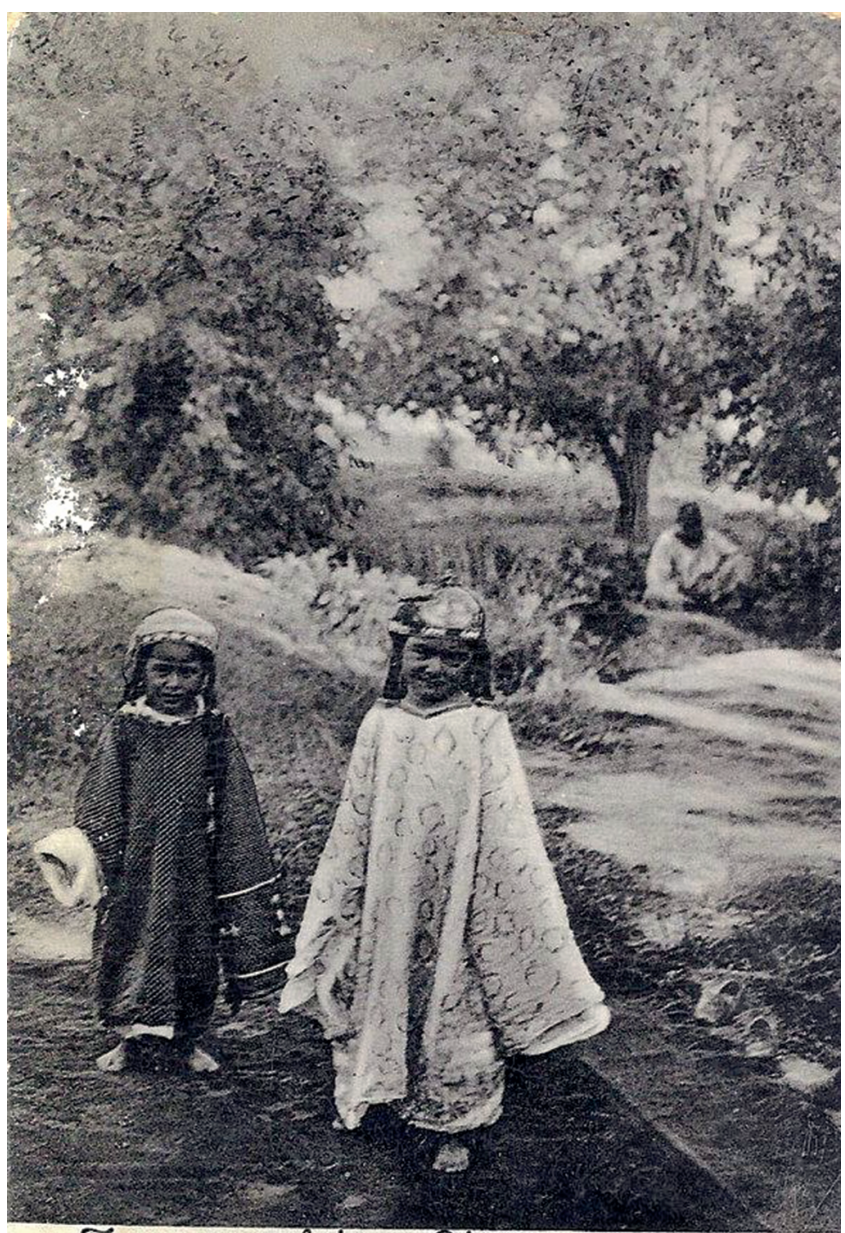

Jung capmobckuxz Dtmeü.

Abbildung 11: Russische Ansichtskarte «Tipy sartovskikh Detej», dt.: „Typen von sartischen Kindern“.

88 Aus der Sammlung von Nizami Ibraimov, Moskau. 
Diese „Haupthandlung“ auf der Ansichtskarte ist als nichts anderes als ein „fait social total“ (eine „totale soziale Tatsache“) $\mathrm{zu}$ betrachten. Unter Bezugnahme auf Marcel Mauss ${ }^{89}$ überträgt Bourdieu diesen Begriff auch auf seine Bildquelle (Manets Gemälde „Frühstück im Grünen“), wenn er feststellt, dass „... in jedem sozialen Fakt das ganze System von Beziehungen enthalten ist, das die betreffende Gesellschaft konstituiert ... "90 In diese Richtung weist auch die Bildüberschrift „Typen von sartischen Kindern“ hin, wenn wir uns die Bedeutung vergegenwärtigen, die der Begriff „Typ“, „Typus“ damals hatte: „»Unter >Typ< verstehen wir das Beispiel für irgendeine natürliche Gruppe, das alle charakteristischen Merkmale dieser Gruppe besitzt.«"91

Bourdieu präzisiert die Inhalte der „totalen sozialen Tatsache“: Dazu gehören im Falle von „Frühstück im Grünen“ nicht nur der spezifische künstlerische Bereich, sondern auch die Politik, die kulturelle Ordnung, die staatliche Ordnung sowie auch die Dispositionen des Künstlers. Auf die betrachtete Ansichtskarte übertragen, heisst es, dass neben den erwähnten Inhalten auch die Kindheit der damaligen Zeit en miniature auf ihre Vorderseite transportiert und somit für die historische Analyse zugänglich gemacht wurde. Woran wird dies erkennbar?

\section{Vier Merkmale einer anderen Kindheit}

Eine entscheidende Hilfe leistet nun die zweite theoretische Einbindung, nämlich die Theorie von Philippe Ariès über die Kindheit in der traditionalen und Industriegesellschaft. In seinem Buch thematisiert oder, genauer gesagt, konstruiert Ariès einen Gegensatz zwischen der traditionalen (traditionsgebundenen) und der Industriegesellschaft in Europa am Beispiel einiger für diese Gesellschaften charakteristischen Einstellungen zum Kind und zum Kindesalter.

Die traditionale Gesellschaft hätte, so der Autor, vom Kind und mehr noch vom Heranwachsenden nur schwach entwickelte Vorstellungen gehabt, während in der Industriegesellschaft die Kindheit als spezifisches Lebensalter mit entsprechenden sozialen Praktiken sehr genau definiert und etabliert ist. Dieser Unterschied hatte nach Ariès vielfältige Folgen in Bezug auf das Kind und die Kindheit in der tradionalen resp. Industriegesellschaft:

Erstens: Die Dauer der Kindheit in der traditionalen Gesellschaft war sehr kurz und beschränkte sich nur auf den Zeitraum, in dem sich das Kind ohne

89 Mauss 1975: 9-144.

90 Bourdieu 2015: 169.

91 Daston/Galison 2002: 53. 
fremde Hilfe (sprich: seiner Eltern und nächsten Verwandten) nicht zurechtfinden konnte. ${ }^{92}$ In der Industriegesellschaft wird die Kindheit jedoch zeitlich ausgedehnt und inkludiert nicht nur die Kindheit selbst, sondern auch die Periode des Heranwachsens;

Zweitens: Sobald das Kind in der tradionalen Gesellschaft physisch imstande war, sich ohne fremde Hilfe in der Welt zu bewegen, wurde es „übergangslos“ in die Erwachsenenwelt integriert, indem es vor allem deren Arbeit und Unterhaltung (bei Ariès: „Spiele“) teilen musste. Die Industriegesellschaft nimmt sich im Gegensatz dazu reichlich Zeit und baut zahlreiche Barrieren, bevor sie „ihre“ Kinder vollständig in das erwachsene Universum aufnimmt;

Drittens: Die Weitergabe der Werte und der Kenntnisse, die Erziehung und allgemein die Sozialisation des Kindes wurden in der traditionalen Gesellschaft von seiner Familie weder gewährleistet noch kontrolliert. Dies geschah nach der Meinung von Ariès hauptsächlich durch das Lehrverhältnis dank einem nicht separierten Zusammenleben des Kindes bzw. des Jugendlichen mit Erwachsenen. Der Autor bringt es auf den Punkt, wenn er sagt: „Es lernte Dinge, die es wissen mußte, indem es den Erwachsenen bei ihrer Verrichtung half.“93 Es war also, wie wir heute sagen würden, ein „learning by doing“. Die Industriegesellschaft etabliert dagegen eine spezifische Institution für die besagte Weitergabe der Werte und der Kenntnisse, nämlich die Schule;

Viertens: Ariès attestiert der traditionalen Gesellschaft geradezu eine fehlende Empathie bzw. eine Gefühls- und Emotionsarmut in Bezug auf das Kind. Das Kind konnte in seinen „allerersten Jahren“ auf „eine oberflächliche Gefühlszuwendung rechnen“, die der Autor leicht ironisch „Gehätschel“ nennt. ${ }^{94}$ Diese Gefühlsarmut sieht der Historiker in den damaligen gesellschaftlichen Verhältnissen und dem entsprechenden Typus der alten Familie begründet: „Die Mission, von der diese alte Familie geprägt war, war die Erhaltung des Besitzes, die gemeinsame Ausübung eines Handwerks, die alltägliche gegenseitige Hilfe in einer Welt, in der ein einzelner Mann und mehr noch eine einzelne Frau nicht überleben konnten, und im Krisenfalle die Verteidigung von Ehre und Leben. Eine affektive Funktion hatte sie nicht ... “95 Die Liebe $\mathrm{zu}$ den Kindern, so wie wir sie kennen, stellt sich erst in der Industriegesellschaft ein.

92 Nalivkin merkt zu der kurzen Dauer der Kindheit im damaligen Mittelasien an: „Sobald das Kind aus dem Gröbsten heraus ist, hört die Mutter in erheblichem Maße auf, sich um es zu kümmern ... “ Abašin 2015: 324.

93 Ariès 1998: 45-46.

94 Ariès 1998: 46.

95 Ariès 1998: 47. 
Allerdings stellt Ariès die Existenz der Liebe und anderer Gefühle zwischen Eheleuten, zwischen Eltern und Kindern in der traditionalen Gesellschaft nicht in Abrede. Er betont nur, dass die Gefühle „keine unabdingbare Voraussetzung für die Existenz wie für das Gleichgewicht der Familie“ waren. Und er schliesst den Satz mit den Worten ab: „... um so besser, wenn sie sich zusätzlich einstellten.“96

Aufgrund dieser Ausführungen von Ariès lassen sich vier gegensätzliche praxeologische Merkmale konstruieren, welche für die Analyse der historischen Ansichtskarten zum Thema Kindheit in Russisch-Mittelasien angewandt werden könnten:

Erstes Merkmal: Missachtung der Morphologie des Kindes in der traditionalen Gesellschaft versus Rücksicht auf die spezifische Konstitution des Kindes in der Industriegesellschaft. Dieses Merkmal findet vor allem in den alltäglichen Praktiken des Kleidens und Bekleidens des Kindes seinen Niederschlag. Denn in diesen lassen sich nicht nur geschlechts-, alters- und klassenspezifische und andere Merkmale einer Gesellschaft beobachten, sondern es treten auch „tiefer gelegene“ Praktiken, wie z. B. Praktiken der Kindheit, zu Tage;

Zweites Merkmal: Weitergabe der Werte und der Kenntnisse durch das Lehrverhältnis in der traditionalen Gesellschaft versus Weitergabe durch eine spezifische Institution (die Schule) in der Industriegesellschaft.

Drittes Merkmal: Recht frühe Integration des Kindes in die Welt der Erwachsenen, direkt nachdem es sich selbständig bewegen kann versus recht behutsame und zeitlich sehr ausgedehnte Heranführung des Kindes in die industrielle Arbeitswelt der Erwachsenen;

Viertes Merkmal: Nicht-Trennung der Welt des Kindes von der der Erwachsenen in der traditionalen Gesellschaft versus Schaffung von sehr kindspezifischen Welten (Krippen, Kindergärten, Spielplätze, Kinder- und Jugendorganisationen etc.) in der Industriegesellschaft.

Was wird aus den historischen Ansichtskarten ersichtlich, wendet man diese vier Merkmale auf ihre Analyse an?

In Bezug auf das erste Merkmal (Morphologie des Kindes) findet sich bei Ariès folgende Beobachtung: „... An der Kleidung zeigt sich, wie wenig die Kindheit in der Lebenspraxis damals (in der traditionalen Gesellschaft - B.S.) als solche behandelt wurde. Sobald das Kind den Windeln, d. h. der Stoffbahn, die man ihm eng um den Körper wickelte, entwuchs, wurde es wie die anderen Männer und Frauen seines Standes gekleidet." "97 In Turkestan musste das Kind in die ihm zugewiesene Kleidung der Erwachsenen, welche in der Tat alterslos

96 Ariès 1998: 47.

97 Ariès 1998: 112. 
war, hineinwachsen, während es heutzutage seiner kindspezifischen Kleidung entwächst, selbst wenn diese - in ärmeren Familien - von seinen älteren Geschwistern oder gar von einem Elternteil stammt. Im letzteren Fall handelt es sich in meisten Fällen um eine umgeänderte und an die Morphologie des Kindes angepasste Kleidung. Wie sehr sich die Kindheit als soziale Praxis gewandelt hat, lässt sich am Streit in der Familie erkennen, zu dem es oft wegen der Übergabe der Kleidung von älteren an jüngere Geschwister kommt. Für die damaligen Verhältnisse jedoch trifft voll und ganz auch die folgende Anmerkung von Ariès zu, wie aus der Ansichtskarte „Samarkand. Mečet’ „ŠahiZinda“. U mogily. № 27“ /dt.: Samarkand. Moschee Schahi-Sinda. Neben einem Grab. Nr.7“98 ersichtlich wird (siehe Abbildung 12): „... Hinsichtlich seines Aufzuges unterschied sich das Kind in nichts vom Erwachsenen. Eine gegensätzlichere Haltung in bezug auf die Kindheit läßt sich nicht denken.“99 Auf der Ansichtskarte ist ein Ausflug von Kindern dokumentiert, die wie Erwachsene en miniature gekleidet sind und dem heutigen Betrachter wie Zwerge vorkommen.

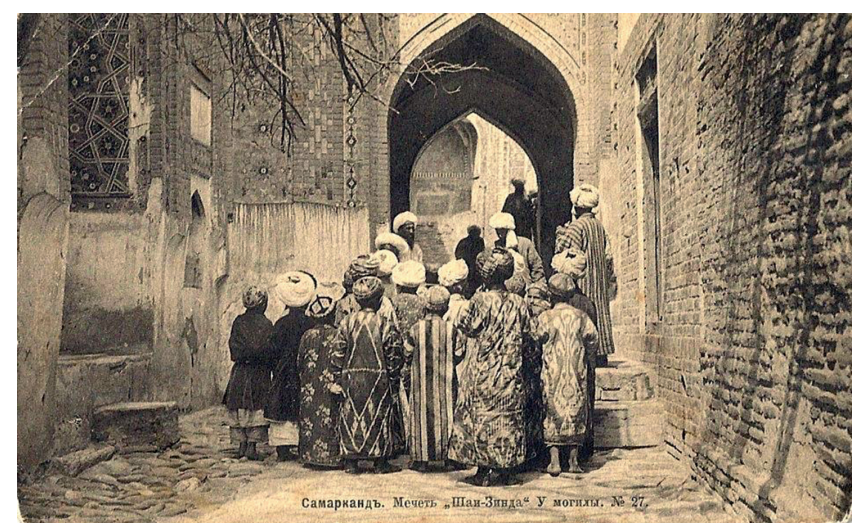

Abbildung 12: Russische Ansichtskarte "Samarkand. Mečet’ „Šahi- Zinda“. U mogily. No 27», dt.: Samarkand. Moschee Schahi Sinda. Neben einem 10 Grab. Nr.7.

In Bezug auf das zweite Merkmal (Lehrverhältnis versus Schule) herrscht in der gegenwärtigen Zentralasienforschung nach wie vor ein Missverständnis. Die damals vorhandene Institution, die unter dem Namen „Maktab“ („Mektebe“)

98 Aus der Sammmlung von Nizami Ibraimov, Moskau.

99 Ariès 1998: 112. 
bekannt ist, ${ }^{100}$ wird fälschlicherweise als „Schule“ übersetzt. Sie war jedoch nur dem Namen nach eine Schule; in Wirklichkeit handelte es sich dabei um ein persönliches Lehrverhältnis oder regelmässige Repetitorien. (siehe Abbildung 13 «518 Vidy Turkestana. Za urokom gramoty», dt.: „518 Ansichten von Turkestan. Während eines Alphabetisierungskurses“). ${ }^{101}$ Ausserdem fand der Unterricht in vielen Fällen nicht ganzjährig, sondern meistens im Winter oder im frühen Sommer statt. Es gab dort keine Differenzierung nach dem jeweiligen Alter; jüngere Schüler wurden oft nicht nur vom Lehrer, sondern auch von älteren „Semestern“ unterrichtet. Über diese Art von „Schulen“ schrieb Constantin Graf von der Pahlen Folgendes: „Die Jungen wachsen unter den Männern heran. Früh nimmt der Vater den Sohn mit, wenn er ausreitet oder in die Stadt geht. Weit entfernt von ihrem Haus begegnet man unterwegs oft solchen Jungen, die mit dem Vater auf einem Pferd reiten. Vom sechsten Lebensjahr an beginnt für den Sohn die Zeit, in der jeder Familieneinfluß aufhört. Das ist der Eintritt in die Schule, die Mechtebe oder tatarisch Maktaba ... “102 „... Wenn der kleine Junge in die Mechtebe eintritt, findet er andere Jungen vor, die schon mehrere Jahre dort sitzen und immer dasselbe auswendig gelernt haben und immer wieder lernen und lesen müssen: das heilige Gesetz des Propheten, den Shariat ... “. ${ }^{103}$

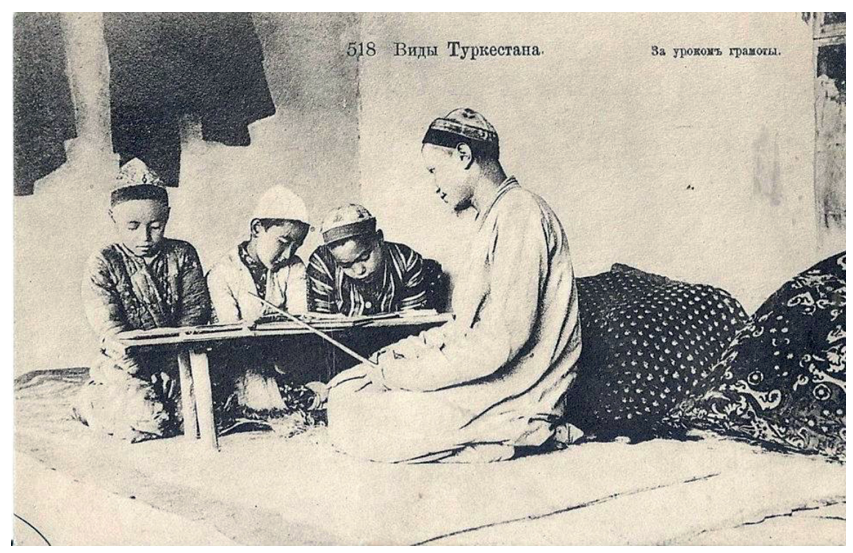

Abbildung 13: Russische Ansichtskarte «518 Vidy Turkestana. Za urokom gramoty», dt.: „518 Ansichten von Turkestan. Während eines Alphabetisierungskurses“.

100 Bendrikov 1960: 36-48. Ausführlicher zu den Unterrichtsinhalten eines städtischen Maktab siehe Pahlen 1969: 65-77.

101 Aus der Sammmlung von Nizami Ibraimov, Moskau.

102 Pahlen 1969: 63.

103 Pahlen 1969: 64. 
Das dritte Merkmal (recht frühe Integration von Kindern in die Arbeitswelt) ist ebenfalls oft zu erkennen, z. B. auf der Ansichtskarte „Types de l'Asie centrale. Forge du pays“ (siehe Abbildung 14) oder auf der Ansichtskarte „Kokand. Kindermusikanten“ (siehe Abbildung 15): ${ }^{104}$ „... Einmal waren die Kinder im alltäglichen Leben mitten unter den Erwachsenen, und an allen alltäglichen Anlässen wie der Arbeit, dem müßigen Umherschlendern oder auch dem Spiel nahmen Kinder und Erwachsene gemeinsam teil ... “105

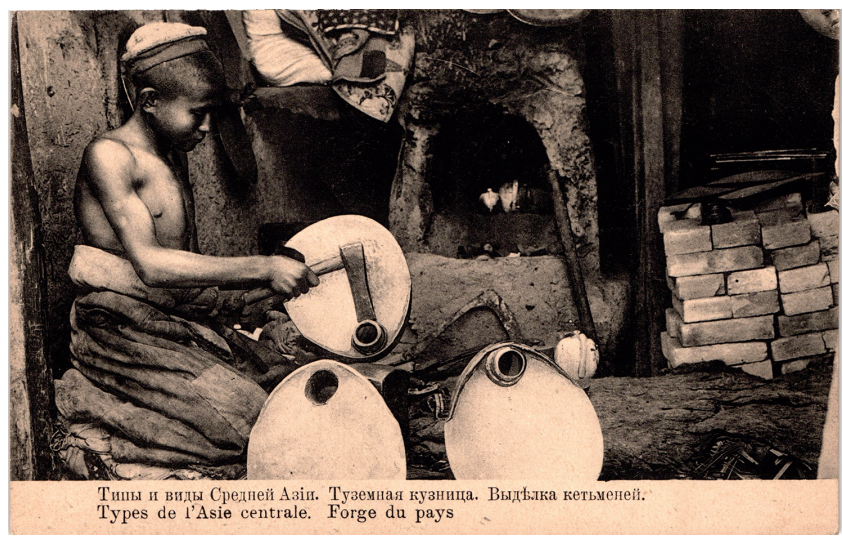

Abbildung 14: Russische Ansichtskarte «Types de l'Asie centrale. Forge du pays».

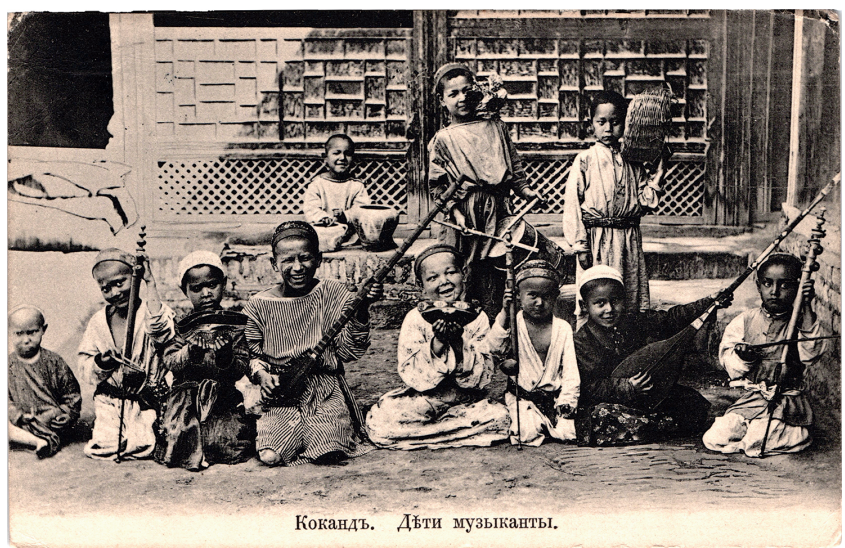

Abbildung 15: Russische Ansichtskarte «Kokand. Deti muzykanty», dt.: „Kokand. Kindermusikanten.“.

104 Aus der Ansichtskartensammlung des Autors.

105 Ariès 1998: 24. 
Das vierte Merkmal (Nicht-Trennung der Welt des Kindes von der der Erwachsenen) ist ebenfalls aus der betrachteten Ansichtskarte („Typen der sartischen Kinder") ersichtlich: Die Eltern bzw. Verwandten verrichten ihre Arbeit auf dem Feld bzw. im Garten und ihre Kinder sind mit dabei. Diese Nicht-Trennung dürfte allumfassend gewesen sein: Es findet sich keine einzige Ansichtskarte oder Fotografie, die vagabundierende Kinder-Bettler zeigt, denn selbst die ärmsten Kinder standen ausschliesslich unter der Obhut ihrer Verwandtschaft.

\section{Schlussfolgerungen}

Die erste Analysestufe diente vor allem dem Zweck a) der Aufhebung der „bedeutungslosen Bedeutung“ der Fotografie auf der Vorderseite der Ansichtskarte und b) ihrer Überführung in eine „lesbare“ Quelle, d.h. ein historisches Dokument. Dies gelingt durch eine bedeutungsstiftende Analysearbeit mit der Ansichtskarte und ihrem Produzenten, dem Fotografen. Die Ansichtskarte ist dadurch keine blosse Fotografie mehr, die eine „bedeutungslose Bedeutung“ hat, sondern ein Produkt, ein Werk, welches aus einer Beziehung zwischen dem Habitus des Fotografen und dem sozialen Feld der Orientfotografie hervorgegangen ist. Deswegen war es erforderlich, sowohl den Habitus des Fotografen wie auch die Genese der russischen Orientfotografie zu beschreiben.

Des Weiteren ist die historische Ansichtskarte eine kontextualisierte und in Form einer Ansichtskarte produzierte Fotografie, die durch ihre performative Notwendigkeit eine Bedeutung erhält: „Wozu wurde diese Fotografie als eine Ansichtskarte herausgegeben?“ Diese performative Notwendigkeit hebt die „,bedeutungslose Bedeutung“ der Fotografie auf, lädt $\mathrm{zu}$ einer bestimmten Betrachtungsweise ein und holt den Betrachter aus seiner passiven Haltung heraus.

Die Auffassung der Ansichtskarte als ein Produkt aus einer Beziehung zwischen dem Habitus des Fotografen und dem Feld der Orientfotografie erlaubt uns auch deren Verbindungen zum Aufbau und der Struktur der Fotografie auf der Vorderseite der Ansichtskarte herzustellen. Dadurch werden potenzielle historische Inhalte $\mathrm{zu}$ Tage gefördert, die auf der zweiten Analysestufe dank der praxeologischen „Lektüre“ zum Sprechen gebracht werden können. Aus der praxeologisch angelegten Analyse von Bildpostkarten wird deutlich, dass uns in diesen sowohl die russischen Praktiken des Sehens als auch unterschiedliche Spuren der historischen Wirklichkeit im damaligen Mittelasien begegnen. 
Die durch die Analyse des Habitus des Fotografen freigelegten Parallelen zwischen der russischen Ansichtskarte und der europäischen Malerei (Darstellung von Kindern im Vorder- versus Erwachsenen im Hintergrund, als eine Gruppe, in welcher eines der Kinder zur Handlung erklärenden Hauptfigur gemacht und so dargestellt wird) sind weder ein Zufall noch eine willkürliche Interpretation, sondern in der Genese des Feldes der russischen Orientfotografie angelegt. Sie erlauben einen Rückschluss auf den Habitus des Fotografen. Selbst wenn der Fotograf der betrachteten Ansichtskarte mit der europäischen Malerei nicht vertraut war, trug er ihre Spuren in seinem Habitus bzw. in seinem technisch, fotografisch und kulturell „geschulten“ Auge.

Die zweite Analysestufe ermöglichte durch ein Strukturierungsangebot (Praxeologisierung), sowohl die visuellen Erfahrungen von der analysierten Ansichtskarte $\mathrm{zu}$ ordnen wie auch beim heutigen Betrachter einen adäquaten kognitiven Stil zu schaffen. Die Betrachtung der Ansichtskarte in ihrer doppelläufigen Hermeneutik erlaubt es, die sozialen Praktiken auf den beiden Seiten der damaligen russischen Gesellschaft $\mathrm{zu}$ analysieren: zum einen die russisch-europäische Sicht, d.h. die visuellen Praktiken der Metropole, als auch die Spuren der historischen Wirklichkeit in Mittelasien, d.h. die Alltagspraktiken der lokalen Bevölkerung.

In der dritten Analysestufe wurde die praxistheoretische Einbindung der visuellen Quelle durch eine Theorie aus der Feder des französischen Historikers Philippe Ariès ergänzt, welche das „ganz Grosse“ (die „totale soziale Tatsache“) erklärte.

Die vorgestellten praxistheoretischen Vorgehensweisen könnten es uns ermöglichen, eine „andere“ Geschichte der Kindheit im Spannungsfeld der zaristischen Ordnungsbildung des Sozialen von Zentrum und Peripherie in Angriff zu nehmen. Doch damit entwerfen wir ein nächstes Feld, welches auf ein anderes grundlegendes Forschungsdesiderat aufmerksam macht.

Danksagung: Für wohlwollende Kritik und fruchtbaren Austausch danke ich: Dr. Birte Kleine-Benne, Erwin Liedke (beide Berlin), Prof. Dr. Anke von Kügelgen (Bern), Dr. Mareike Beez (Erfurt), Dr. Marcus Held (Mannheim), Prof. Dr. Sergej Abašin, Nizami Ibraimov (beide Moskau).

\section{Bibliographie}

Abašin, Sergej (2007): Die Sartenproblematik in der russischen Geschichtsschreibung des 19. und des ersten Viertels des 20. Jahrhunderts. Übers. von Marcus Held. Berlin: Schwarz. 
Abašin, Sergej (Hrsg.) (2015): Polveka v Turkestane. V. P. Nalivkin: biografija, dokumenty, trudy (Ein halbes Jahrhundert in Turkestan. V. P. Nalivkin: Biographie, Dokumente, Werke). Moskau: Izdatel'skiy dom Mardžani.

Agamben, Giorgio (1979): Infanzia e storia: distruzione dell'esperienza e origine della storia. Torino: G. Einaudi.

Agamben, Giorgio (1993): Infancy and History: The Destruction of Experience. London; New York: Verso.

Ajni, Sadriddin (1950): Raby (Sklaven). Moskva: Sovetskij pisatel'.

Ajni, Sadriddin (1952): Buchara: Vospominanija (Buchara: Erinnerungen). Moskva: Sovetskij pisatel'.

Antweiler, Christoph (2013): 1914 - Welt in Farbe: Farbfotografie vor dem Krieg. Ostfildern: Hatje Cantz.

Ariès, Philippe (1998): Geschichte der Kindheit. München: Deutscher Taschenbuch Verlag. Banks, Marcus / Vokes, Richard (2015): “Introduction: Anthropology, Photography and the Archive". History and Anthropology 21.4: 337-349.

Barto, Agnija (1949): Stikhi detjam (Gedichte für Kinder). Moskau, Leningrad: Detgiz.

Baumgarten, Elisheva (2004): Mothers and children: Jewish family life in medieval Europe. Princeton, NJ: Princeton University Press.

Baxandall, Michael (1980): Die Wirklichkeit der Bilder: Malerei und Erfahrung im Italien des 15. Jahrhunderts. Übers. von Hans Günther Holl. Frankfurt am Main: Syndikat-Verl.

Bendrikov, Kiriak (1960): Očerki po istorii narodnogo obrazovanija v Turkestane (1865-1924)

(Überblick über die Volksbildung in Turkestan 1865-1924). Moskau: Akademija pedagogičeskich nauk RSFSR.

Berkner, L. K. (1973): "Recent Research on the History of the Family in Europe”. Journal of Marriage and Family 35: 395-405.

Betscher, Silke (2014): „Bildsprache. Möglichkeiten und Grenzen einer Visuellen Diskursanalyse“. In: Bilder in historischen Diskursen. Hrsg. von Franz X. Eder. Wiesbaden: Springer VS, 63-83.

Bourdieu, Pierre (2006): Eine illegitime Kunst: die sozialen Gebrauchsweisen der Photographie. Hamburg: Europäische Verl.-Anst.

Bourdieu, Pierre (2013): Die männliche Herrschaft. Frankfurt am Main: Suhrkamp.

Bourdieu, Pierre (2014): Über den Staat: Vorlesungen am Collège de France; 1989-1992. Übers. von Horst Brühmann und Petra Willim. Berlin: Suhrkamp.

Bourdieu, Pierre (2015): Manet: eine symbolische Revolution; Vorlesungen am Collège de France 1998-2000. Übers. von Achim Russer und Bernd Schwibs. Berlin: Suhrkamp.

Brodskaja, Dina (1938): Marijkino detstvo (Die Kindheit von Marijka). Leningrad: Detizdat.

Bruštejn, Aleksandra (1956): Doroga uchodit vdal' (Der Weg führt in die Ferne). Moskau: Detizdat.

Cassirer, Ernst (1997): Philosophie der symbolischen Formen. Darmstadt: Primus.

Daston, Lorraine / Galison, Peter (2002): „Das Bild der Objektivität “. In: Ordnungen der Sichtbarkeit: Fotografie in Wissenschaft, Kunst und Technologie. Hrsg. von Peter Geimer. Frankfurt am Main: Suhrkamp, 29-99.

Demaitre, Luke (1976-1977): “The Idea of Childhood and Childcare in the Medical Writings of the Middle Ages". History of Childhood Quarterly 4: 461-490.

DeMause, Lloyd (1974): The History of Childhood. New York: Psychohistory Press.

Derenthal, Ludger (2012): Das koloniale Auge: frühe Porträtfotografie in Indien. Leipzig: Koehler \& Amelang. 
Dlužnevskaja, Galina (2011): Archeologičeskie issledovanija v Central'noj Azii i Sibiri 1859-1959 gg. (Archäologische Forschungen in Zentralasien und Sibirien in den Jahren 1859-1859). St.-Petersburg: ElekSis.

Etkind, Aleksandr (2011): Internal Colonization: Russia's Imperial Experience. Cambridge: Polity. Geimer, Peter (2009): Ordnungen der Sichtbarkeit: Fotografie in Wissenschaft, Kunst und Technologie. Frankfurt am Main: Suhrkamp.

Geimer, Peter (2014): Theorien der Fotografie zur Einführung. Hamburg: Junius.

Gugutzer, Robert (2015): Soziologie des Körpers. Bielefeld: transcript.

Gurevič, Aron (2009): Individuum i socium na srednevekom Zapade (Mensch und Gesellschaft im mittelalterlichen Europa). St.-Petersburg: ALEXANDRIA.

Habermas, Rebekka (1998): "Parent-Child Relationships in the 19th Century”. German History 16.1: 43-55.

Herlihy, David (1985): Medieval Households. Cambridge, MA: Harvard University Press. Hermann, Rainer (2015): Tausendundein Traum. http://www.faz.net/aktuell/reise/ausstellungin-konstanz-tausendundein-traum-13447969.html?printPagedArticle = true (13.10.2016).

Holmes, Urban T. (1968-1969): “Medieval Childhood”. Journal of Social History 2: 164-172. Inber, Vera (1954): Kak ja byla malen'koj (Als ich klein war). Moskau: Detgiz.

Isaacson, Joel / Bouillon, Jean Paul (1980): The Crisis of Impressionism, 1878-1882. Ann Arbor, MI: The Museum of Art.

Isakovskij, Mikhail (1956): Sočinenija $v$ dvukh tomakh (Gesammelte Werke in 2 Bänden). Moskau: Khudožestvennaja literatura.

Iser, Wolfgang (1994): Der Akt des Lesens: Theorie ästhetischer Wirkung. München: Fink. Kassil', Lev A. (1935): Konduit i Švambranija (Konduit und Švambranija). Moskva: Detizdat. Latour, Bruno (2007): Eine neue Soziologie für eine neue Gesellschaft: Einführung in die AkteurNetzwerk-Theorie. Übers. von Gustav Roßler. Frankfurt am Main: Suhrkamp.

Margolina, Sonja (1992): Das Ende der Lügen: Russland und die Juden im 20. Jahrhundert. Berlin: Siedler.

Mauss, Marcel (1975): Soziologie und Anthropologie. München, Wien: Hanser.

Mclaughlin, Mary (1974): "Survivors and Surrogates: Children and Parents from the 9th to the 13th Centuries". In: The History of Childhood. Edited by Lloyd deMause. New York: Psychohistory Press, 101-181.

Pahlen, Constantin (1969): Im Auftrag des Zaren in Turkestan: 1908-1909. Stuttgart: Steingrüben.

Pollock, Linda A. (1983): Forgotten Children: Parent-Child Relations from 1500 to 1900. Cambridge: Cambridge Univeristy Press.

Priščepova, Valerija (2007): “Central'naja Azija v fotografijakh Rossijskich issledovatelej” (Zentralasien-Fotografien der russischen Forscher). Central'naja Azija: tradicija $v$ uslovijakh peremen Vyp. I: 223-226.

Priščepova, Valerija (2011a): Illustrativnye kollektsii po narodam Tsentral'noj Azii vtoroj poloviny XIX - načala XX veka $v$ sobranijach Kunstkamery (Visuelle Archive zu Völkern Zentralasiens aus der zweiten Hälfte des 19. - Anfang des 20. Jhs. in Beständen der Kunstkamera). St.Petersburg: Nauka.

Priščepova, Valerija (2011b): “K 150-letiju so dnja roždenija S. M. Dudina - chudožnika, etnografa” (Zum 150. Geburtstag des Malers und Ethnographen S. M. Dudin). Antropologičeskij Forum 15: 608-649.

Rakhimov, Rakhmat (1998): Detstvo $v$ tradicionnoj kul'ture narodov Srednej Azii, Kazakhstana $i$ Kavkaza. St.-Peterburg: MAE RAN. 
Šanijazov, Karim / Ismailov, Khajot (1981): Etnografičeskie očerki material'noj kultury uzbekov konca XIX - načala XX v. (Ethnographischer Überblick über materielle Kultur der Usbeken Ende des 19. - Anfang des 20 Jhs.). Taschkent: Fan.

Schatzki, Theodore R. (2002): The Site of the Social: A Philosophical Account of the Constitution of Social Life and Change. University Park, PA.: Pennsylvania State Univ. Press.

Schmidt, Robert (2008): Symbolische Gewalt: Herrschaftsanalyse nach Pierre Bourdieu. Konstanz: UVK-Verl.-Ges.

Schmidt, Robert (2012): Soziologie der Praktiken: konzeptionelle Studien und empirische Analysen. Berlin: Suhrkamp.

Shorter, Edward (1975): The Making of the Modern Family. New York: Basic Books.

Sommer, Manfred (1999): Sammeln: ein philosophischer Versuch. Frankfurt am Main: Suhrkamp.

Stiegler, Bernd / Thürlemann, Felix (2015): Orientbilder: Fotografien 1850-1910. Frankfurt am Main: Weissbooks.

Stone, Lawrence (1977): The Family, Sex and Marriage in England 1500-1800. London: Weidenfeld \& Nicolson.

Sukhareva, Ol'ga (1979): Kostjum narodov Srednej Azii: Istoriko-etnografičeskie očerki (Völkertrachten in Mittelasien: Historisch-etnographischer Überblick). Moskva: Nauka.

Sukhareva, Ol'ga (1982): Istorija sredneaziatskogo kostjuma: Samarkand, 2-ja polovina $X I X$ - načalo XX v. (Geschichte der mittelasiatischen Tracht: Samarkand, 2. Hälfte des 19. Anfang des 20 Jhs.). Moskva: Nauka.

Uspenskij, Anton (2016): “Samuil Dudin. Vospitanie glaza” (Samuil Dudin. Erziehung des Auges). In: Turkestan: Moi mir islama. Hrsg. von Efim Rezvan. St.-Petersburg / Almaty: KMBCH, 162-183.

Vokes, Richard (2015): “Reflections on a Complex (and Cosmopolitan) Archive: Postcards and Photography in Early Colonial Uganda, c. 1904-1928”. History and Anthropology 21:4: 375-409.

Zittel, Claus (2014): „Die Ordnung der Diskurse und das Chaos der Bilder. Bilder als blinde Flecken in Foucaults Diskursanalyse und in der Historiographie der Philosophie?“. In: Bilder in historischen Diskursen. Hrsg. von Franz X. Eder. Wiesbaden: Springer VS, 85-107. 